\title{
Classification of graded Hecke algebras for complex reflection groups
}

\author{
Arun Ram and Anne V. Shepler
}

\begin{abstract}
The graded Hecke algebra for a finite Weyl group is intimately related to the geometry of the Springer correspondence. A construction of Drinfeld produces an analogue of a graded Hecke algebra for any finite subgroup of $G L(V)$. This paper classifies all the algebras obtained by applying Drinfeld's construction to complex reflection groups. By giving explicit (though nontrivial) isomorphisms, we show that the graded Hecke algebras for finite real reflection groups constructed by Lusztig are all isomorphic to algebras obtained by Drinfeld's construction. The classification shows that there exist algebras obtained from Drinfeld's construction which are not graded Hecke algebras as defined by Lusztig for real as well as complex reflection groups.
\end{abstract}

Mathematics Subject Classification (2000). 20C, 20F, 52B.

Keywords. Reflection group, Coxeter group, Weyl group, affine Hecke algebra, Iwahori-Hecke algebra, representation theory, graded Hecke algebra.

\section{Introduction}

This paper is motivated by a general effort to generalize the theory of Weyl groups and their relation to groups of Lie type to the setting of complex reflection groups. One natural question is whether there are affine Hecke algebras corresponding to complex reflection groups. If they exist then it might be possible to use these algebras to build an analogue of the Springer correspondence for complex reflection groups.

A priori, one knows how to construct affine Hecke algebras corresponding only to Weyl groups since both a finite real reflection group $W$ and a $W$-invariant lattice (the existence of which forces $W$ to be a Weyl group) are needed in the construction. Our search for analogues of graded Hecke algebras for complex reflection groups was motivated by Lusztig's results [Lu2] showing that the geometric

Research of the first author supported in part by the National Security Agency and by EPSRC Grant GR K99015 at the Newton Institute for Mathematical Sciences. Research of the second author supported in part by National Science Foundation grant DMS-9971099. 
information contained in the affine Hecke algebra can be recovered from the corresponding graded Hecke algebra. Lusztig [Lu] defines the graded Hecke algebra for a finite Weyl group $W$ with reflection representation $V$. Let $t_{g}, g \in W$, be a basis for the group algebra $\mathbb{C} W$ of $W$ and let $k_{\alpha} \in \mathbb{C}$ be "parameters" indexed by the roots in the root system of $W$ such that $k_{\alpha}$ depends only on the length of the root $\alpha$. Then the graded Hecke algebra $H_{\mathrm{gr}}$ depending on the parameters $k_{\alpha}$ is the (unique) algebra structure on $S(V) \otimes \mathbb{C} W$ such that

(a) the symmetric algebra of $V, S(V)=S(V) \otimes 1$, is a subalgebra of $H_{\mathrm{gr}}$,

(b) the group algebra $\mathbb{C} W=1 \otimes \mathbb{C} W=\operatorname{span}-\left\{1 \otimes t_{g} \mid g \in W\right\}$ is a subalgebra of $H_{\mathrm{gr}}$, and

(c) $t_{s_{i}} v=\left(s_{i} v\right) t_{s_{i}}-k_{\alpha_{i}}\left\langle v, \alpha_{i}^{\vee}\right\rangle$ for all $v \in V$ and simple reflections $s_{i}$ in the simple roots $\alpha_{i}$.

This definition applies to all finite real reflection groups $W$ since the simple roots and simple reflections are well defined. Unfortunately, the need for simple reflections in the construction makes it unclear how to define analogues for complex reflection groups.

For finite real reflection groups, the graded Hecke algebra $H_{\text {gr }}$ is a "semidirect product" of the polynomial ring $S(V)$ and the group algebra $\mathbb{C} W$. Drinfeld [Dr] defines a different type of semidirect product of $S(V)$ and $\mathbb{C} W$, and Drinfeld's construction applies to all finite subgroups $G$ of $G L(V)$. In this paper, we

(1) classify all the algebras obtained by applying Drinfeld's construction to finite complex reflection groups $G$,

(2) show that every graded Hecke algebra $H_{\text {gr }}$ (as defined by Lusztig) for a finite real reflection group is isomorphic to an algebra obtained by Drinfeld's construction by giving explicit isomorphisms between these algebras.

The results from (2) show how Drinfeld's construction is a true generalization of Lusztig's construction of graded Hecke algebras, something which is not obvious. Our classification in (1) gives a complete solution to the problem of finding all graded Hecke algebras for finite reflection groups.

A consequence of our classification is that there exist graded Hecke algebras for finite real reflection groups which are not obtained with Lusztig's construction. In this sense, Drinfeld's construction is a strict generalization of the algebras $H_{\mathrm{gr}}$. These new algebras, and the algebras corresponding to complex reflection groups that are not real reflection groups, deserve further study and probably have interesting representation theories.

For us, one surprising result of our classification is that no nontrivial graded Hecke algebra structures exist for many complex reflection groups. In some sense, this is disappointing, as we would have liked to find nontrivial and interesting structures for each complex reflection group.

It might be that we have not yet hit upon the "right" definition of graded Hecke algebras. For example, we show that there do not exist nontrivial graded Hecke algebra structures, according to Drinfeld's definition, for any of the complex reflection groups $G(r, 1, n)=(\mathbb{Z} / r \mathbb{Z}) \imath S_{n}$ when $r>2$ and $n>3$. On the other 
hand, in the last section of this paper we are able to construct algebras that "look" like they ought to be graded Hecke algebras corresponding to these groups. Is it possible that there is a "better" definition of graded Hecke algebras which applies to complex reflection groups and which includes the algebras that we introduce in Section 5 as examples?

Acknowledgements. We thank C. Kriloff for numerous stimulating conversations during our work on this paper. A. Ram thanks the Newton Institute for the Mathematical Sciences at Cambridge University for hospitality and support (EPSRC Grant No. GR K99015) during Spring 2001 when the writing of this paper was completed.

\section{Graded Hecke algebras}

In this section, we define the graded Hecke algebra following Drinfeld [Dr]. Our main result in this section is Theorem $1.9 \mathrm{c}$, which determines exactly how many degrees of freedom one has in defining a graded Hecke algebra.

Let $V$ be an $n$ dimensional vector space over $\mathbb{C}$ and let $G$ be a finite subgroup of $G L(V)$. The group algebra of $G$ is

$$
\mathbb{C} G=\mathbb{C}-\operatorname{span}\left\{t_{g} \mid g \in G\right\}, \quad \text { with } \quad t_{g} t_{h}=t_{g h} .
$$

Let $a_{g}: V \times V \longrightarrow \mathbb{C}$ be skew symmetric bilinear forms indexed by the elements of $G$ and let $A$ be the associative algebra generated by $V$ and $\mathbb{C} G$ with the additional relations

$$
t_{h} v t_{h^{-1}}=h v \quad \text { and } \quad[v, w]=\sum_{g \in G} a_{g}(v, w) t_{g}, \quad \text { for } h \in G \text { and } v, w \in V
$$

where $[v, w]=v w-w v$. These relations allow every element $a \in A$ to be written in the form

$$
a=\sum_{g \in G} p_{g} t_{g}, \quad p_{g} \in S(V),
$$

where $S(V)$ is the symmetric algebra of $V$. More precisely, one must fix a section of the canonical surjection $T(V) \rightarrow S(V)$ from the tensor algebra of $V$ to $S(V)$ and take the elements $p_{g}$ to be in the image of this section.

The structure of $A$ depends on the choices of the "parameters" $a_{g}(v, w) \in \mathbb{C}$. Our goal is to determine when the algebra $A$ will be a "semidirect product" of $S(V)$ and $\mathbb{C} G$. This idea motivates the following definition [Dr].

The algebra $A$ is a graded Hecke algebra for $G$ if

$$
A \cong S(V) \otimes \mathbb{C} G \quad \text { as a vector space }
$$


or, equivalently, if the expression in (1.2) is unique for each $a \in A$. A general element $a \in A$ is a linear combination of products of elements $t_{g}$ and $u_{i}$, where $\left\{u_{1}, u_{2}, \ldots, u_{n}\right\}$ is a basis of $V$. There are two straightening operations needed to put $a$ in the form (1.2):

(a) moving $t_{h}$ 's to the right, and (b) putting $u_{i} u_{j}$ pairs in the correct order.

These two straightening operations correspond to the two identities in (1.1). Note that the "correct order" of $u_{i} u_{j}$ is determined by the choice of the section of the projection $T(V) \rightarrow S(V)$. Let $v_{1}, v_{2}, v_{3}$ be arbitrary elements of $V$ and let $h \in G$. Applying the straightening operations to $t_{h} v_{1} v_{2}$ gives

$$
\begin{aligned}
t_{h} v_{1} v_{2} & =t_{h}\left[v_{1}, v_{2}\right]+t_{h} v_{2} v_{1} & & \text { (rearrange } \left.v_{1} \text { and } v_{2}\right) \\
& =t_{h}\left[v_{1}, v_{2}\right]+\left(h v_{2}\right)\left(h v_{1}\right) t_{h} & & \left(\text { move } t_{h}\right. \text { to the right) }
\end{aligned}
$$

and applying the straightening operations in a different order gives

$$
\begin{aligned}
t_{h} v_{1} v_{2} & =\left(h v_{1}\right)\left(h v_{2}\right) t_{h} \\
& =\left[h v_{1}, h v_{2}\right] t_{h}+\left(h v_{2}\right)\left(h v_{1}\right) t_{h} . \quad \text { (move } t_{h} \text { to the right) }
\end{aligned}
$$

Setting these two expressions equal gives the relation

$$
t_{h}\left[v_{1}, v_{2}\right] t_{h^{-1}}=\left[h v_{1}, h v_{2}\right], \quad \text { for all } h \in G, v_{1}, v_{2} \in V .
$$

Similarly, applying the straightening operations to $v_{1} v_{2} v_{3}$ gives

$$
\begin{array}{rlrl}
v_{1} v_{2} v_{3} & =\left[v_{1}, v_{2}\right] v_{3}+v_{2} v_{1} v_{3} & & \text { (moving } \left.v_{1} \text { past } v_{2}\right) \\
& =\left[v_{1}, v_{2}\right] v_{3}+v_{2}\left[v_{1}, v_{3}\right]+v_{2} v_{3} v_{1} & \text { (moving } \left.v_{1} \text { past } v_{3}\right) \\
& =\left[v_{1}, v_{2}\right] v_{3}+v_{2}\left[v_{1}, v_{3}\right]+\left[v_{2}, v_{3}\right] v_{1}+v_{3} v_{2} v_{1} & \text { (straightening } \left.v_{2} \text { and } v_{3}\right),
\end{array}
$$

and applying the straightening operations in a different order gives

$$
\begin{aligned}
v_{1} v_{2} v_{3} & =v_{1}\left[v_{2}, v_{3}\right]+v_{1} v_{3} v_{2} & & \text { (moving } \left.v_{2} \text { past } v_{3}\right) \\
& =v_{1}\left[v_{2}, v_{3}\right]+\left[v_{1}, v_{3}\right] v_{2}+v_{3} v_{1} v_{2} & & \text { (moving } \left.v_{1} \text { past } v_{3}\right) \\
& =v_{1}\left[v_{2}, v_{3}\right]+\left[v_{1}, v_{3}\right] v_{2}+v_{3}\left[v_{1}, v_{2}\right]+v_{3} v_{2} v_{1} & & \text { (straightening } \left.v_{1} \text { and } v_{2}\right) .
\end{aligned}
$$

These are equal if

$$
\left[v_{1},\left[v_{2}, v_{3}\right]\right]+\left[v_{2},\left[v_{3}, v_{1}\right]\right]+\left[v_{3},\left[v_{1}, v_{2}\right]\right]=0, \quad \text { for all } v_{1}, v_{2}, v_{3} \in V \text {. }
$$

Conversely, the identities (1.3) and (1.4) are exactly what is needed to guarantee that any order of application of the straightening operations (a) and (b) will produce the same normal form (1.2) for the element $a$. Thus we have

Lemma 1.5. Let $A$ be an algebra defined as in (1.1). Then $A$ is a graded Hecke algebra if and only if the identities (1.3) and (1.4) hold in $A$. 
Using (1.1), the relations (1.3) and (1.4) can be rewritten in terms of the bilinear forms $a_{g}: V \times V \rightarrow \mathbb{C}$ as

$$
\begin{gathered}
a_{g}\left(v_{1}, v_{2}\right)=a_{h g h^{-1}}\left(h v_{1}, h v_{2}\right) \quad \text { and } \\
a_{g}\left(v_{3}, v_{1}\right)\left(g v_{2}-v_{2}\right)+a_{g}\left(v_{2}, v_{3}\right)\left(g v_{1}-v_{1}\right)+a_{g}\left(v_{1}, v_{2}\right)\left(g v_{3}-v_{3}\right)=0
\end{gathered}
$$

for $v_{1}, v_{2}, v_{3} \in V$ and $g, h \in G$.

Let $\langle\rangle:, V \times V \rightarrow \mathbb{C}$ be a $G$-invariant nondegenerate Hermitian form on $V$. For each $g \in G$, set

$$
\begin{aligned}
V^{g} & =\{v \in V \mid g v=v\}, \\
\left(V^{g}\right)^{\perp} & =\left\{v \in V \mid\langle v, w\rangle=0 \text { for all } w \in V^{g}\right\}, \quad \text { and } \\
\operatorname{ker} a_{g} & =\left\{v \in V \mid a_{g}(v, w)=0 \text { for all } w \in V\right\} .
\end{aligned}
$$

Lemma 1.8. Let $G$ be a finite subgroup of $G L(V)$ and let $g \in G$.

(a) $\left(V^{g}\right)^{\perp}=\{v-g v \mid v \in V\}$.

(b) Suppose $g \neq 1$. If $\operatorname{codim}\left(V^{g}\right)=2$ and $a: V \times V \rightarrow \mathbb{C}$ is a skew symmetric bilinear form such that ker $a=V^{g}$, then a satisfies (1.7).

Let $A$ be a graded Hecke algebra for $G$ defined by skew symmetric bilinear forms $a_{g}: V \times V \rightarrow \mathbb{C}$.

(c) If $g \neq 1$ then ker $a_{g} \supseteq V^{g}$.

(d) If $g \neq 1$ and $a_{g} \neq 0$ then $\operatorname{ker} a_{g}=V^{g}$ and $\operatorname{codim}\left(V^{g}\right)=2$.

(e) If $g \neq 1$ and $a_{g} \neq 0$ then, for all $h \in G$,

$$
a_{h^{-1} g h}\left(b_{1}, b_{2}\right)=\operatorname{det}\left(h^{\perp}\right) a_{g}\left(b_{1}, b_{2}\right),
$$

where $\left\{b_{1}, b_{2}\right\}$ is a basis of $\left(V^{g}\right)^{\perp}$ and $h^{\perp}:\left(V^{g}\right)^{\perp} \rightarrow\left(V^{g}\right)^{\perp}$ is the composition of $h$ restricted to $\left(V^{g}\right)^{\perp}$ with the canonical projection $V \rightarrow V / V^{g}$.

Proof. (a) Consider the map $\phi: V \rightarrow V$ given by $\phi(v)=v-g v$. Then $\operatorname{ker} \phi=V^{g}$ and $\operatorname{im} \phi \subseteq\left(V^{g}\right)^{\perp}$ since, if $v \in V, w \in V^{g}$, then

$$
\langle v-g v, w\rangle=\langle v, w\rangle-\langle g v, w\rangle=\langle v, w\rangle-\langle g v, g w\rangle=\langle v, w\rangle-\langle v, w\rangle .
$$

Since $\operatorname{dim}(\operatorname{im} \phi)=\operatorname{codim}(\operatorname{ker} \phi)=\operatorname{codim}\left(V^{g}\right)$ it follows that $\operatorname{im} \phi=\left(V^{g}\right)^{\perp}$.

(b) Let $v_{1}, v_{2}, v_{3} \in V$. If any $v_{i} \in V^{g}$, then (1.7) holds trivially for the skew symmetric form $a$. So assume each $v_{i} \notin V^{g}$ and write each $v_{i}$ as $v_{i}^{+}+v_{i}^{-}$where $v_{i}^{+} \in V^{g}$ and $v_{i}^{-} \in\left(V^{g}\right)^{\perp}$. Then

$$
a\left(v_{i}, v_{j}\right)=a\left(v_{i}^{-}, v_{j}^{-}\right) \quad \text { and } \quad v_{i}-g v_{i}=v_{i}^{-}-g v_{i}^{-} .
$$

Since $\operatorname{dim}\left(V^{g}\right)^{\perp}=2$, at least one of the $v_{i}^{-}$is a linear combination of the other two. Say $v_{1}^{-}=c_{2} v_{2}^{-}+c_{3} v_{3}^{-}$with $c_{2}, c_{3} \in \mathbb{C}$. Substituting $v_{i}-g v_{i}=v_{i}^{-}-g v_{i}^{-}$ and $v_{1}^{-}=c_{2} v_{2}^{-}+c_{3} v_{3}^{-}$then yields

$$
\begin{aligned}
& a\left(v_{3}, v_{1}\right)\left(g v_{2}-v_{2}\right)+a\left(v_{2}, v_{3}\right)\left(g v_{1}-v_{1}\right)+a\left(v_{1}, v_{2}\right)\left(g v_{3}-v_{3}\right) \\
& \quad=a\left(v_{3}^{-}, v_{1}^{-}\right)\left(g v_{2}^{-}-v_{2}^{-}\right)+a\left(v_{2}^{-}, v_{3}^{-}\right)\left(g v_{1}^{-}-v_{1}^{-}\right)+a\left(v_{1}^{-}, v_{2}^{-}\right)\left(g v_{3}^{-}-v_{3}^{-}\right)=0,
\end{aligned}
$$


and so (1.7) holds.

(c) Let $v_{3} \in V^{g}$ and $v_{2} \in V$.

If $v_{2} \in V^{g}$, then $a_{g}\left(v_{2}, v_{3}\right)\left(g v_{1}-v_{1}\right)=0$ for all $v_{1} \in V$ by (1.7). Since $V^{g} \neq V$, there is some $v_{1}$ such that $g v_{1} \neq v_{1}$ and so $a_{g}\left(v_{2}, v_{3}\right)=0$.

If $v_{2} \notin V^{g}$, let $v_{1}=\sum_{k=1}^{r-1} g^{k} v_{2}$, where $r$ is the order of $g$. By $(1.6), a_{g}\left(v_{3}, g^{k} v_{2}\right)=$ $a_{g}\left(g^{-k} v_{3}, v_{2}\right)=a_{g}\left(v_{3}, v_{2}\right)$, for any $k$, and so

$$
\begin{aligned}
0 & =a_{g}\left(v_{3}, v_{1}\right)\left(g v_{2}-v_{2}\right)+a_{g}\left(v_{2}, v_{3}\right)\left(g v_{1}-v_{1}\right) \\
& =(r-1) a_{g}\left(v_{3}, v_{2}\right)\left(g v_{2}-v_{2}\right)+a_{g}\left(v_{3}, v_{2}\right)\left(g v_{2}-v_{2}\right)=r a_{g}\left(v_{3}, v_{2}\right)\left(g v_{2}-v_{2}\right) .
\end{aligned}
$$

Thus $a_{g}\left(v_{3}, v_{2}\right)=0$. Hence, for all $v_{2} \in V, a_{g}\left(v_{3}, v_{2}\right)=0$ and so $V^{g} \subseteq \operatorname{ker} a_{g}$.

(d) By (c), $\operatorname{codim}\left(V^{g}\right) \geq \operatorname{codim}\left(\operatorname{ker} a_{g}\right)$. Since $a_{g} \neq 0$, there exist $v, w \in V$ with $a_{g}(v, w) \neq 0$ and so $\operatorname{codim}\left(\operatorname{ker} a_{g}\right) \geq 2$. Let $v_{1}-g v_{1}$ and $v_{2}-g v_{2}$ be linearly independent elements of $\left(V^{g}\right)^{\perp}$. Then (1.7) implies that any element $v_{3}-g v_{3} \in\left(V^{g}\right)^{\perp}$ is a linear combination of $v_{1}-g v_{1}$ and $v_{2}-g v_{2}$, and so

$$
2 \geq \operatorname{dim}\left(\left(V^{g}\right)^{\perp}\right)=\operatorname{codim}\left(V^{g}\right) \geq \operatorname{codim}\left(\operatorname{ker} a_{g}\right) \geq 2 .
$$

Thus $V^{g}=\operatorname{ker} a_{g}$ and $\operatorname{codim}\left(V^{g}\right)=2$.

(e) Write $h b_{1}=h_{11} b_{1}+h_{21} b_{2}+\left(h b_{1}\right)^{g}$ and $h b_{2}=h_{12} b_{1}+h_{22} b_{2}+\left(h b_{2}\right)^{g}$ with $h_{i j} \in \mathbb{C}$ and $\left(h b_{i}\right)^{g} \in V^{g}$. Then

$$
\begin{aligned}
a_{h^{-1} g h}\left(b_{1}, b_{2}\right) & =a_{g}\left(h b_{1}, h b_{2}\right) \\
& =a_{g}\left(h_{11} b_{1}+h_{21} b_{2}+\left(h b_{1}\right)^{g}, h_{12} b_{1}+h_{22} b_{2}+\left(h b_{2}\right)^{g}\right) \\
& =\left(h_{11} h_{22}-h_{21} h_{12}\right) a_{g}\left(b_{1}, b_{2}\right)=\operatorname{det}\left(h^{\perp}\right) a_{g}\left(b_{1}, b_{2}\right)
\end{aligned}
$$

since $a_{g}$ is skew symmetric and $V^{g} \subseteq \operatorname{ker} a_{g}$.

The following theorem is a slightly strengthened version of statements (given without proof) in $[\mathrm{Dr}]$.

Theorem 1.9. Let $G$ be a finite subgroup of $G L(V)$ and let $Z_{G}(g)=\{h \in$ $G \mid h g=g h\}$ denote the centralizer of an element $g$ in $G$.

(a) If $A$ is a graded Hecke algebra for $G$, then the values of $a_{h^{-1} g h}$ are determined by the values of $a_{g}$ via the equation

$$
a_{h^{-1} g h}\left(v_{1}, v_{2}\right)=a_{g}\left(h v_{1}, h v_{2}\right), \quad \text { for all } g, h \in G, v_{1}, v_{2} \in V \text {. }
$$

(b) For $g \neq 1$, there is a graded Hecke algebra $A$ with $a_{g} \neq 0$ if and only if $\operatorname{ker} a_{g}=V^{g}, \quad \operatorname{codim}\left(V^{g}\right)=2, \quad$ and $\quad \operatorname{det}\left(h^{\perp}\right)=1$, for all $h \in Z_{G}(g)$, 
where $h^{\perp}$ is $h$ restricted to the space $\left(V^{g}\right)^{\perp}$. In this case, $a_{g}$ is determined by its value $a_{g}\left(b_{1}, b_{2}\right)$ on a basis $\left\{b_{1}, b_{2}\right\}$ of $\left(V^{g}\right)^{\perp}$.

(c) Let $d$ be the number of conjugacy classes of $g \in G$ such that $\operatorname{codim}\left(V^{g}\right)=2$ and $\operatorname{det}\left(h^{\perp}\right)=1$ for all $h \in Z_{G}(g)$, where $h^{\perp}$ is $h$ restricted to the space $\left(V^{g}\right)^{\perp}$. The sets $\left\{a_{g}\right\}_{g \in G}$ corresponding to graded Hecke algebras $A$ form a vector space of dimension $d+\operatorname{dim}\left(\left(\bigwedge^{2} V\right)^{G}\right)$.

Proof. (a) is simply a restatement of (1.6).

(b) $\Longrightarrow$ : If $A$ is a graded Hecke algebra and $a_{g} \neq 0$ then by Lemma $1.8 \mathrm{~d}$, $\operatorname{codim}\left(V^{g}\right)=2$ and $\operatorname{ker} a_{g}=V^{g}$. So $a_{g}$ is determined by its value $a_{g}\left(b_{1}, b_{2}\right)$ on a basis $b_{1}, b_{2}$ of $\left(V^{g}\right)^{\perp}$. Suppose $h \in Z_{G}(g)$. Then, by Lemma 1.8e,

$$
a_{g}\left(b_{1}, b_{2}\right)=a_{h g h^{-1}}\left(h b_{1}, h b_{2}\right)=a_{g}\left(h b_{1}, h b_{2}\right)=\operatorname{det}\left(h^{\perp}\right) a_{g}\left(b_{1}, b_{2}\right),
$$

and so $\operatorname{det}\left(h^{\perp}\right)=1$. Note that $h\left(V^{g}\right)=V^{g}$ and $h\left(V^{g}\right)^{\perp}=\left(V^{g}\right)^{\perp}$ since, for each $v \in V^{g}, h(v)=h g(v)=g h(v)$.

$\Longleftarrow$ : If $\operatorname{codim}\left(V^{g}\right)=2$ then, up to constant multiples, there is a unique skew symmetric form on $V$ which is nondegenerate on $\left(V^{g}\right)^{\perp}$ and which has ker $a_{g}=V^{g}$. Fix such a form and then define forms $a_{h}, h \in G$, by

$$
a_{h}\left(v_{1}, v_{2}\right)= \begin{cases}a_{g}\left(k v_{1}, k v_{2}\right) & \text { if } h=k^{-1} g k \\ 0 & \text { otherwise }\end{cases}
$$

for $v_{1}, v_{2} \in V$. Let $a_{1}$ be any $G$-invariant skew symmetric form on $V$. Then this collection $\left\{a_{g}\right\}_{g \in G}$ of skew symmetric bilinear forms satisfies (1.6) by definition and (1.7) by Lemma 1.8b. Thus (by Lemma 1.5), it determines a graded Hecke algebra $A$ via (1.1).

(c) From (a) and (b) it follows that the sets $\left\{a_{g}\right\}_{g \in G}$, running over all graded Hecke algebras $A$ for $G$, form a vector space. Since each of the collections $\left\{a_{g}\right\}_{g \neq 1}$ constructed by (1.10) has its support on a single conjugacy class, these collections form a basis of the vector space of sets $\left\{a_{g}\right\}_{g \neq 1}$. The only condition on the form $a_{1}$ is that it satisfies (1.6), which means that it is a $G$-invariant element of $\left(\bigwedge^{2} V\right)^{*}$.

The following consequence of Theorem 1.9 will be useful for completing the classification of graded Hecke algebras for complex reflection groups.

Corollary 1.11. Assume that $G$ contains $h=\xi \cdot 1$ for some $\xi \in \mathbb{C} \backslash\{ \pm 1\}$. If $A$ is a graded Hecke algebra for $G$, then $a_{g}=0$ for all $g \neq 1$.

Proof. If $h=\xi \cdot 1 \in G$, then $h \in Z_{G}(g)$ for every $g \in G$ and $\operatorname{det}\left(h^{\perp}\right)=\xi^{2}$ if $\operatorname{codim}\left(V^{g}\right)=2$. The statement then follows from Theorem 1.9b. 


\section{The classification for reflection groups}

A reflection is an element of $G L(V)$ that has exactly one eigenvalue not equal to 1. The reflecting hyperplane of a reflection is the $(n-1)$-dimensional subspace which is fixed pointwise. A complex reflection group $G$ is a finite subgroup of $G L(V)$ generated by reflections. The group $G$ is irreducible if $V$ cannot be written in the form $V=V_{1} \oplus V_{2}$ where $V_{1}$ and $V_{2}$ are $G$-invariant subspaces. The group $G$ is a real reflection group if $V=\mathbb{C} \otimes_{\mathbb{R}} V_{\mathbb{R}}$ for a real vector space $V_{\mathbb{R}}$ and $G \subseteq G L\left(V_{\mathbb{R}}\right)$.

The following facts about reflection groups are well known.

Lemma 2.1. Let $G$ be an irreducible reflection group.

(a) [ST, Theorem 5.3] The number of elements $g \in G$ such that $\operatorname{codim}\left(V^{g}\right)=2$ is $\sum_{i<j} m_{i} m_{j}$ where $m_{1}, \ldots, m_{n}$ are the exponents of $G$.

(b) [Ca, Lemma 2] If $G$ is a real reflection group and $g \in G$ with $\operatorname{codim}\left(V^{g}\right)=2$, then $g$ is the product of two reflections.

(c) [OT, Theorem 6.27] For any $g \in G$, the space $V^{g}$ is the intersection of reflecting hyperplanes.

Remark. The statement of Lemma 2.1b does not hold for complex reflection groups. Consider the exceptional complex reflection group $G_{4}$ of rank 2 , in the notation of Shephard and Todd [ST]. All the reflections have order 3 and $-1 \in G_{4}$. Suppose $-1=r s$ for two reflections $r$ and $s$. If $s$ has eigenvalues 1 and $\omega$, where $\omega$ is a primitive cube root of unity, then $r^{-1}=-s$ has eigenvalues -1 and $-\omega$, a contradiction to the assumption that $r$ is a reflection. Thus $-1 \in G_{4}$ is not a product of two reflections.

Lemma 2.2. Let $G \subseteq G L(V)$ be a complex reflection group. Let $A$ be a graded Hecke algebra for $G$ and let $g \in G$. Let $V^{G}=\{v \in V \mid g v=v$ for all $g \in G\}$ be the invariants in $V$.

(a) If $g=1$ and $\operatorname{dim} V^{G} \leq 1$, then $a_{g}=0$.

(b) If the order of $g$ is 2 , then $a_{g}=0$.

Proof. (a) Let $\langle\rangle:, V \times V \rightarrow \mathbb{C}$ be a nondegenerate $G$-invariant Hermitian form on $V$ and write $V=V^{G} \oplus\left(V^{G}\right)^{\perp}$ where $\left(V^{G}\right)^{\perp}=\left\{v \in V \mid\langle v, w\rangle=0\right.$ for all $\left.w \in V^{G}\right\}$. Since $\operatorname{dim}\left(V^{G}\right) \leq 1$ and $a_{1}$ is skew symmetric, $a_{1}$ restricted to $V^{G}$ is 0 . There is a basis $\alpha_{1}, \ldots, \alpha_{k}$ of $\left(V^{G}\right)^{\perp}$ and constants $\xi_{1}, \ldots, \xi_{k} \in \mathbb{C}, \xi_{i} \neq 1$, such that the reflections $s_{1}, \ldots, s_{k}$ given by

$$
s_{i} v=v+\left(\xi_{i}-1\right) \frac{\left\langle v, \alpha_{i}\right\rangle}{\left\langle\alpha_{i}, \alpha_{i}\right\rangle} \alpha_{i}, \quad \text { for } v \in V,
$$

are in $G$. Equation (1.6) implies that, for any $v \in V$,

$$
a_{1}\left(\alpha_{i}, v\right)=a_{1}\left(s_{i} \alpha_{i}, s_{i} v\right)=a_{1}\left(\xi_{i} \alpha_{i}, v+\left(\xi_{i}-1\right) \frac{\left\langle v, \alpha_{i}\right\rangle}{\left\langle\alpha_{i}, \alpha_{i}\right\rangle} \alpha_{i}\right)=\xi_{i} a_{1}\left(\alpha_{i}, v\right),
$$


since $a_{1}\left(\alpha_{i}, \alpha_{i}\right)=0$ (as $a_{1}$ is skew symmetric). Since $\xi_{i} \neq 1, a_{1}\left(\alpha_{i}, v\right)=0$ for $1 \leq i \leq k$. Thus $\operatorname{ker} a_{1}=V$.

(b) Since $g^{2}=1$, all eigenvalues of $g$ are \pm 1 . If $\operatorname{codim}\left(V^{g}\right) \neq 2$, then $a_{g}=0$ by Theorem 1.9b. If $\operatorname{codim}\left(V^{g}\right)=2$, then

$$
g=\mathrm{id}_{V^{g}} \oplus\left(-\mathrm{id}_{\left(V^{g}\right)^{\perp}}\right)
$$

as a linear transformation on $V$. By [St1, Theorem 1.5], [Bou V, $\S 5$ Ex. 8], the stabilizer, $\operatorname{Stab}\left(V^{g}\right)$, of $V^{g}$ is a reflection subgroup of $G$ and so there is a reflection $s \in \operatorname{Stab}\left(V^{g}\right)$ that is the identity on $V^{g}$. So $s \in Z_{G}(g)$ and $\operatorname{det}(s)=\operatorname{det}\left(s^{\perp}\right) \neq 1$, where $s^{\perp}$ is $s$ restricted to $\left(V^{g}\right)^{\perp}$. Thus, by Theorem $1.9 \mathrm{~b}, a_{g}=0$.

\section{A. Real reflection groups}

If $G \subseteq G L(V)$ is a real reflection group then $V=\mathbb{C} \otimes_{\mathbb{R}} V_{\mathbb{R}}$ and $G \subseteq G L\left(V_{\mathbb{R}}\right)$, where $V_{\mathbb{R}}$ is a real vector space. We shall assume that $G$ is irreducible.

Let us recall some basic facts about real reflection groups which can be found in $[\mathrm{Hu}]$ or [Bou]. The action of $G$ on $V_{\mathbb{R}}$ has fundamental chambers $w C$ indexed by $w \in G$. The roots for $G$ are vectors $\alpha \in V_{\mathbb{R}}$ such that the reflections in $G$ are the reflections $s_{\alpha}$ in the hyperplanes

$$
H_{\alpha}=\left\{v \in V_{\mathbb{R}} \mid\langle v, \alpha\rangle=0\right\} .
$$

For each fundamental chamber $C$, the reflections $s_{1}, s_{2}, \ldots, s_{n}$ in the hyperplanes $H_{\alpha_{1}}, H_{\alpha_{2}}, \ldots, H_{\alpha_{n}}$ that bound $C$ form a set of simple reflections for $G$. The simple reflections obtained from a different choice of fundamental chamber $w C$ are $w s_{1} w^{-1}, \ldots, w s_{n} w^{-1}$.

Theorem 2.3. Let $G \subseteq G L\left(V_{\mathbb{R}}\right)$ be a real reflection group. Let $s_{1}, \ldots, s_{n}$ be a set of simple reflections in $G$ and let $m_{i j}$ be the order of $s_{i} s_{j}$. Then $g \in G$ satisfies $g^{2} \neq 1, \operatorname{codim}\left(V^{g}\right)=2$, and $\operatorname{det}\left(h^{\perp}\right)=1$ for all $h \in Z_{G}(g)$ (the conditions in Theorem 1.9c) if and only if $g$ is conjugate to

$$
\left(s_{i} s_{j}\right)^{k}, \quad \text { with } 0<k<\frac{m_{i j}}{2},
$$

for some $1 \leq i, j \leq n$.

Proof. $\Longrightarrow$ : Let $\alpha$ and $\beta$ be two roots such that $V^{g}=H_{\alpha} \cap H_{\beta}$ (see Lemma 2.1c). Then $H_{\alpha} \cap H_{\beta}$ has nontrivial intersection with some fundamental chamber $C$ for $W$, and we may assume that $H_{\alpha}$ and $H_{\beta}$ are walls of the chamber $C$ (since $C$ is a cone in $\mathbb{R}^{n}$ ). Since choosing simple reflections with respect to a different chamber $w C$ corresponds to conjugation by $w$, we may assume that the reflections in the hyperplanes $H_{\alpha}$ and $H_{\beta}$ are simple reflections and $\alpha=\alpha_{1}$ and $\beta=\alpha_{2}$. 
The element $g$ is an element of the stabilizer $\operatorname{Stab}\left(V^{g}\right)$, which is a reflection group by [St1, Theorem 1.5]. Since $\operatorname{codim}\left(V^{g}\right)=2, \operatorname{Stab}\left(V^{g}\right)$ is a rank two real reflection group, and therefore a dihedral group. This dihedral group is generated by the two simple reflections $s_{1}$ and $s_{2}$ in the hyperplanes $H_{\alpha_{1}}$ and $H_{\alpha_{2}}$ (restricted to $\left.\left(V^{g}\right)^{\perp}\right)$ and all reflections have determinant -1 . Let $g^{\perp}$ be the element $g$ restricted to $\left(V^{g}\right)^{\perp}$. Since $g \in Z_{G}(g)$, $\operatorname{det}\left(g^{\perp}\right)=1$, and so $g$ must be a product of an even number of reflections. Thus $g=\left(s_{1} s_{2}\right)^{k}$ or $g=\left(s_{2} s_{1}\right)^{k}$, for some $0<k \leq m / 2$, where $m$ is the order of $s_{1} s_{2}$. Since $g^{2} \neq 1, k \neq m / 2$, and so $g$ is conjugate to $\left(s_{1} s_{2}\right)^{k}$ for some $0<k<m / 2$.

$\Longleftarrow$ : Assume that $g=\left(s_{i} s_{j}\right)^{k}$ for some $0<k<m_{i j} / 2$. Then $V^{g}=H_{\alpha_{i}} \cap H_{\alpha_{j}}$ and so $\operatorname{codim}\left(V^{g}\right)=2$. Since $g$ is a product of an even number of reflections, $\operatorname{det}\left(g^{\perp}\right)=1$. The only elements of $O\left(V_{\mathbb{R}}\right) \cong O_{2}(\mathbb{R})$ that are diagonalizable in $G L\left(V_{\mathbb{R}}\right) \cong G L_{2}(\mathbb{R})$ are \pm 1 and elements with determinant -1 . Thus, the eigenvectors of the element $g^{\perp}$ (which has distinct eigenvalues since it is not \pm 1 ) do not lie in $V_{\mathbb{R}}$, only in $V=\mathbb{C} \otimes_{\mathbb{R}} \mathbb{R}$. Let $h \in Z_{G}(g)$ and let $h^{\perp} \in O\left(V_{\mathbb{R}}\right) \cong O_{2}(\mathbb{R})$ denote $h$ restricted to $\left(V^{g}\right)^{\perp}$. Since $h^{\perp}$ commutes with $g^{\perp}$ and $g^{\perp}$ has distinct eigenvalues, $g^{\perp}$ and $h^{\perp}$ have the same eigenvectors. Hence, $\operatorname{det} h^{\perp}=1$.

Using Theorem 2.3 and Theorem 1.9b, we can read off the graded Hecke algebras for the irreducible real reflection groups from the Dynkin diagrams. For each irreducible real reflection group, label a set of simple reflections $s_{1}, \ldots, s_{n}$ using the Dynkin diagrams below. If nodes $i$ and $j$ and nodes $j$ and $k$ are connected by single edges, then $s_{i} s_{j}$ is conjugate to $s_{j} s_{k}$ via the element $s_{i} s_{j} s_{k}$.

The following table gives representatives of the conjugacy classes of $g \in G$ that may have $a_{g} \neq 0$ for some graded Hecke algebra $A$. We assume that the reflection group $G$ is acting on its irreducible reflection representation $V$. When $G$ is the symmetric group $S_{n}$ acting on an $n$-dimensional vector space $V$ by permutation matrices, then $\operatorname{dim}\left(V^{G}\right)=1$ and, by Lemma 2.2a and Theorem 2.3, $a_{g} \neq 0$ for some graded Hecke algebra $A$ only if $g$ is conjugate to the three cycle $(1,2,3)=s_{1} s_{2}$ (this example is analyzed in Section 3).

\begin{tabular}{|l|c|}
\hline Type & $\begin{array}{c}\text { Representative } \\
\text { with } a_{g} \neq 0\end{array}$ \\
\hline$A_{n-1}$ & $s_{1} s_{2}$ \\
$B_{n}$ & $s_{1} s_{2}, s_{2} s_{3}$ \\
$D_{n}$ & $s_{2} s_{3}$ \\
$E_{6}, E_{7}, E_{8}$ & $s_{1} s_{4}$ \\
$F_{4}$ & $s_{1} s_{2}, s_{2} s_{3}, s_{3} s_{4}$ \\
$H_{3}, H_{4}$ & $s_{1} s_{2},\left(s_{1} s_{2}\right)^{2}, s_{2} s_{3}$ \\
$I_{2}(m)$ & $\left(s_{1} s_{2}\right)^{k}, 0<k<m / 2$ \\
\hline
\end{tabular}

Table 1. Graded Hecke algebras for real reflection groups 




Figure 1. Coxeter-Dynkin diagrams for real reflection groups

\section{B. Complex reflection groups $G(r, p, n)$}

The irreducible complex reflection groups were classified by Shephard and Todd [ST]. There is one infinite family denoted $G(r, p, n)$ and a list of exceptional complex reflection groups denoted $G_{4}, \ldots, G_{35}$. In this subsection, we classify the graded Hecke algebras for the groups $G(r, p, n)$.

Let $r, p$ and $n$ be positive integers with $p$ dividing $r$ and let $\xi=e^{2 \pi i / r}$. Let $S_{n}$ be the symmetric group of $n \times n$ matrices and let

$$
\xi_{j}=\operatorname{diag}(1,1, \ldots, 1, \xi, 1, \ldots, 1),
$$

where $\xi$ appears in the $j$ th entry. Then

$$
G(r, p, n)=\left\{\xi_{1}^{\lambda_{1}} \cdots \xi_{n}^{\lambda_{n}} w \mid w \in S_{n}, 0 \leq \lambda_{i} \leq r-1, \lambda_{1}+\cdots+\lambda_{n}=0 \bmod p\right\} .
$$

For $\lambda=\left(\lambda_{1}, \ldots, \lambda_{n}\right) \in(\mathbb{Z} / r \mathbb{Z})^{n}$, let $\xi^{\lambda}=\xi_{1}^{\lambda_{1}} \cdots \xi_{n}^{\lambda_{n}}$. Then the multiplication in $G(r, p, n)$ is described by the relations

$$
\xi^{\lambda} \xi^{\mu}=\xi^{\lambda+\mu} \quad \text { and } \quad w \xi^{\lambda}=\xi^{w \lambda} w, \quad \text { for } \lambda, \mu \in(\mathbb{Z} / r \mathbb{Z})^{n}, w \in S_{n},
$$

where $S_{n}$ acts on $(\mathbb{Z} / r \mathbb{Z})^{n}$ by permuting the factors. Let $v_{i}$ be the column vector with 1 in the $i^{\text {th }}$ entry and all other entries 0 . The group $G(r, p, n)$ acts on $V:=\mathbb{C}^{n}$ with orthonormal basis $\left\{v_{1}, \ldots, v_{n}\right\}$ as a complex reflection group. 
Every real reflection group is a complex reflection group and several of these are special cases of the groups $G(r, p, n)$. In particular,

(a) $G(1,1, n)$ is the symmetric group $S_{n}$,

(b) $G(2,1, n)$ is the Weyl group $W B_{n}$ of type $B_{n}$,

(c) $G(2,2, n)$ is the Weyl group $W D_{n}$ of type $D_{n}$, and

(d) $G(r, r, 2)$ is the dihedral group $I_{2}(r)$ of order $2 r$.

The reflections in $G(r, p, n)$ are

$$
\begin{array}{cc}
\xi_{i}^{k p}, & 1 \leq i \leq n, 0 \leq k \leq(r / p)-1, \text { and } \\
\xi_{i}^{k} \xi_{j}^{-k}(i, j), & 1 \leq i<j \leq n, 0 \leq k \leq r-1,
\end{array}
$$

where $(i, j)$ is the transposition in $S_{n}$ that switches $i$ and $j$.

Conjugacy in $G(r, p, n)$. Each element of $G(r, p, n)$ is conjugate by elements of $S_{n}$ to a disjoint product of cycles of the form

$$
\xi_{i}^{\lambda_{i}} \cdots \xi_{k}^{\lambda_{k}}(i, i+1, \ldots, k) .
$$

By conjugating this cycle by $\xi_{i}^{-c} \xi_{i+1}^{\lambda_{i}} \xi_{i+2}^{\lambda_{i}+\lambda_{i+1}} \cdots \xi_{k}^{\lambda_{i}+\cdots+\lambda_{k-1}} \in G(r, r, n)$, we have $\xi_{i}^{-c} \xi_{k}^{c+\lambda_{i}+\cdots+\lambda_{k}}(i, \ldots, k), \quad$ where $c=(k-i) \lambda_{i}+(k-i-1) \lambda_{i+1}+\cdots+\lambda_{k-1}$.

If $i_{1}, i_{2}, \ldots, i_{\ell}$ denote the minimal indices of the cycles and $c_{1}, \ldots, c_{\ell}$ are the numbers $c$ for the various cycles, then after conjugating by $\xi_{i_{1}}^{c_{1}} \cdots \xi_{i_{\ell-1}}^{c_{\ell-1}} \xi_{i_{\ell}}^{-\left(c_{1}+\cdots+c_{\ell-1}\right)} \in$ $G(r, r, n)$, each cycle becomes

$$
\xi_{k}^{\lambda_{i}+\cdots+\lambda_{k}}(i, \ldots, k) \quad \text { except the last, which is } \quad \xi_{i_{\ell}}^{-a} \xi_{n}^{b}\left(i_{\ell}, \ldots, n\right),
$$

where $a=c_{1}+\cdots+c_{\ell}$ and $b=a+\lambda_{i_{\ell}}+\cdots+\lambda_{n}$. If $k=n-i_{\ell}+1$ is the length of the last cycle, then conjugating the last cycle by $\xi_{i_{\ell}}^{k-1} \xi_{i_{\ell}+1}^{-1} \cdots \xi_{n}^{-1} \in G(r, r, n)$ gives

$$
\xi_{i_{\ell}}^{-a+k} \xi_{n}^{b-k}\left(i_{\ell}, \ldots, n\right)
$$

If we conjugate the last cycle by $\xi_{i_{\ell}}^{p} \in G(r, p, n)$, we have

$$
\xi_{i_{\ell}}^{-a+p} \xi_{n}^{b-p}\left(i_{\ell}, \ldots, n\right)
$$

In summary, any element $g$ of $G(r, p, n)$ is conjugate to a product of disjoint cycles where each cycle is of the form

$$
\xi_{k}^{a}(i, i+1, \ldots, k), \quad 0 \leq a \leq r-1,
$$

except possibly the last cycle, which is of the form

$$
\xi_{i_{\ell}}^{a} \xi_{n}^{b}\left(i_{\ell}, i_{\ell}+1, \ldots, n\right), \quad \text { with } 0 \leq a \leq \operatorname{gcd}(p, k)-1
$$


where $k=n-i_{\ell}+1$ is the length of the last cycle.

Centralizers in $G(r, p, n)$. Let $Z_{G(r, p, n)}(g)=\{h \in G(r, p, n) \mid h g=g h\}$ denote the centralizer of $g \in G(r, p, n)$. Since $G(r, p, n)$ is a subgroup of $G(r, 1, n)$,

$$
Z_{G(r, p, n)}(g)=Z_{G(r, 1, n)}(g) \cap G(r, p, n),
$$

for any element $g \in G(r, p, n)$. Suppose that $g$ is an element of $G(r, 1, n)$ which is a product of disjoint cycles of the form $\xi_{k}^{a}(i, \ldots, k)$ and that $h \in G(r, 1, n)$ commutes with $g$. Conjugation by $h$ effects some combination of the following operations on the cycles of $g$ :

(a) permuting cycles of the same type, $\xi_{k}^{a}(i, \ldots, k)$ and $\xi_{m}^{b}(j, \ldots, m)$ with $b=a$ and $k-i=m-j$,

(b) conjugating a single cycle $\xi_{k}^{a}(i, \ldots, k)$ by powers of itself, and

(c) conjugating a single cycle $\xi_{k}^{a}(i, \ldots, k)$ by $\xi_{i}^{b} \cdots \xi_{k}^{b}$, for any $0 \leq b \leq r-1$.

Furthermore, the elements of $G(r, 1, n)$ which commute with $g$ are determined by how they "rearrange" the cycles of $g$ and a count (see [Mac, p. 170]) of the number of such operations shows that if $g \in G(r, 1, n)$ and $m_{a, k}$ is the number of cycles of type $\xi_{i+k}^{a}(i, i+1, \ldots, i+k)$ for $g$, then

$$
\operatorname{Card}\left(Z_{G(r, 1, n)}(g)\right)=\prod_{a, k}\left(m_{a, k} ! \cdot k^{m_{a, k}} \cdot r^{m_{a, k}}\right) .
$$

Determining the graded Hecke algebras for $G(r, p, n)$. It follows from Lemma 1.8 a that if $g=\xi_{i}^{a+b} \xi_{k}^{-a}(i, \ldots, k)$, then $\left(V^{g}\right)^{\perp}$ has basis

$$
\left\{v_{k}-v_{k-1}, v_{k-1}-v_{k-2}, \ldots, v_{i+1}-\xi^{a} v_{i}\right\} \text { if } b=0, \quad \text { and }\left\{v_{i}, \ldots, v_{k}\right\} \text { if } b \neq 0 .
$$

Thus, if $g \in G(r, p, n)$ and $\operatorname{codim}\left(V^{g}\right)=2$, then $g$ is conjugate to one of the following elements:

$$
\begin{aligned}
& b=\xi_{1}^{a} \xi_{3}^{-a}(1,2,3), \quad 0 \leq a \leq \operatorname{gcd}(p, 3)-1, \\
& c=\xi_{1}^{a+\ell} \xi_{2}^{-a}(1,2), \quad \ell \neq 0(\text { so } r \neq 1), \\
& d=\xi_{1}^{\ell_{1}} \xi_{2}^{\ell_{2}}, \quad \ell_{1} \neq 0, \ell_{2} \neq 0(\text { so } r \neq 1), \\
& e=(1,2) \xi_{3}^{\ell}, \quad \ell \neq 0, \\
& f=(1,2) \xi_{3}^{a} \xi_{4}^{-a}(3,4) .
\end{aligned}
$$

It is interesting to note that these elements are also representatives of the conjugacy classes of elements in $G(r, p, n)$ which can be written as a product of two reflections.

We determine conditions on the above elements and on $r, p$, and $n$ to give nontrivial graded Hecke algebras: 
(z) The center of $G(r, p, n)$ is

$$
Z(G(r, p, n))=\left\{\xi_{1}^{\ell} \cdots \xi_{n}^{\ell} \mid n \ell=0 \bmod p\right\}
$$

Since $\xi_{1}^{p} \cdots \xi_{n}^{p} \in Z(G(r, p, n))$, it follows that $p=r$ or $p=r / 2$ whenever $Z(G(r, p, n)) \subseteq\{ \pm 1\}=\left\{\xi_{1}^{0} \cdots \xi_{n}^{0}, \xi_{1}^{r / 2} \cdots \xi_{n}^{r / 2}\right\}$.

(b1) If $n \geq 4$, the element $\xi_{1} \xi_{2} \xi_{3} \xi_{4}^{-3} \in Z_{G}(b)$ and has determinant $\xi^{2}$ on $\left(V^{b}\right)^{\perp}=$ span- $\left\{v_{3}-v_{2}, v_{2}-\xi^{a} v_{1}\right\}$.

(b2) If $n=3$ and $p=0 \bmod 3$, the element $\xi_{1}^{p / 3} \xi_{2}^{p / 3} \xi_{3}^{p / 3} \in Z_{G}(b)$ and has determinant $\xi^{2 p / 3}$ on $\left(V^{b}\right)^{\perp}$.

(c1) If $n \geq 3$, the element $\xi_{1} \xi_{2} \xi_{3}^{-2} \in Z_{G}(c)$ and has determinant $\xi^{2}$ on $\left(V^{c}\right)^{\perp}=$ $\operatorname{span}-\left\{v_{1}, v_{2}\right\}$.

(c2) If $n=2, p=r / 2$ and $p$ is odd, the element $\xi_{1}^{p / 4} \xi_{2}^{p / 4} \in Z_{G}(c)$ and has determinant $\xi^{r / 2}$ on $\left(V^{c}\right)^{\perp}$.

(d1) If $n \geq 3$, the element $\xi_{1} \xi_{3}^{-1} \in Z_{G}(d)$ and has determinant $\xi$ on $\left(V^{d}\right)^{\perp}=$ $\operatorname{span}-\left\{v_{1}, v_{2}\right\}$.

(d2) If $p=r / 2$, the element $\xi_{1}^{r / 2} \in Z_{G}(d)$ and has determinant $\xi^{r / 2}$ on $\left(V^{d}\right)^{\perp}$.

(ef) The elements $e$ and $f$ have order 2 .

Thus, it follows from Corollary 1.11, Theorem $1.9 \mathrm{~b}$, and Lemma $2.2 \mathrm{~b}$ that if $A$ is a graded Hecke algebra for $G(r, p, n)$, then

$$
\begin{array}{lll}
a_{b}=0 & \text { unless } & \text { (i) } r=1, \text { or } \\
& & \text { (ii) } r=2, \text { or } \\
& & \text { (iii) } n=3 \text { and } p \neq 0 \text { mod } 3, \\
& & \text { (i) } r=2 \text { and } p=1, \text { or } \\
a_{c}=0 & \text { unless } & \text { (ii) } n=2 \text { and } p=r / 2, \\
& & p=r, n=2 \text { and } p \neq 0 \bmod 2, \\
a_{d}=0 & \text { unless } & \text { and } \\
a_{e}=0 & \text { always, } & \\
a_{f}=0 & \text { always. }
\end{array}
$$

In the remaining cases, one uses the description of $Z_{G}(g)$ given just before (2.5) to check that all elements of $Z_{G}(g)$ have determinant 1 on $\left(V^{g}\right)^{\perp}$. Note that $n=3$ and $p \neq 0 \bmod 3$ imply that $a_{b}=0$ for the elements $b=\xi_{1}^{a} \xi_{3}^{-a}(1,2,3)$.

We arrive at the following enumeration of the nontrivial graded Hecke algebras for complex reflection groups. (The tensor product algebra $S(V) \otimes \mathbb{C} G$ always exists and corresponds to the case when all of the skew symmetric forms $a_{g}$ are zero). The table below gives representatives of the conjugacy classes of $g \in G$ that may have $a_{g} \neq 0$ for some graded Hecke algebra $A$. 


\begin{tabular}{|l|c|}
\hline Group & $\begin{array}{c}\text { Representative } g \\
\text { with } a_{g} \neq 0\end{array}$ \\
\hline$G(1,1, n)=S_{n}$ & $(1,2,3)$ \\
$G(2,1, n)=W B_{n}, \quad n \geq 3$ & $\xi_{1}(1,2),(1,2,3)$ \\
$G(2,2, n)=W D_{n}, \quad n \geq 3$ & $(1,2,3)$ \\
$G(r, r, 2)=I_{2}(r)$ & $\xi_{1}^{k} \xi_{2}^{r-k}, 0<k<r / 2$ \\
$G(r, r / 2,2), \quad r / 2$ odd & $\xi_{2}^{r / 2}(1,2)$ \\
$G(r, r, 3), \quad r \neq 0 \bmod 3$ & $(1,2,3)$ \\
$G(r, r / 2,3), \quad r / 2 \neq 0 \bmod 3, r \neq 2$ & $(1,2,3)$ \\
\hline
\end{tabular}

Table 2. Graded Hecke algebras for the groups $G(r, p, n)$

\section{C. Exceptional complex reflection groups}

The irreducible exceptional complex reflection groups $G$ are denoted $G_{4}, \ldots, G_{35}$ in the classification of Shephard and Todd. From Table VII in [ST], one sees that the center of $G$ is \pm 1 only in the cases $G_{4}, G_{12}, G_{24}$ and $G_{33}$. By Schur's lemma, the center of an irreducible complex reflection group consists of multiples of the identity. Thus, by Corollary 1.11, the only exceptional complex reflection groups that could have a nontrivial graded Hecke algebra (i.e., with some $a_{g} \neq 0$ ) are $G_{4}$, $G_{12}, G_{24}$ and $G_{33}$ (we exclude the real groups). We determine the graded Hecke algebras for these groups using Theorem $1.9 \mathrm{~b}$ and Lemma 2.2.

The rank 2 group $G_{4}$ has order 24 and seven conjugacy classes. The following data concerning these conjugacy classes are obtained from the computer software GAP $[\mathrm{S}+]$ using the package CHEVIE $[\mathrm{G}+]$. In the following table, $\omega$ is a primitive cube root of unity and $C(g)$ denotes the conjugacy class of $g$.

\begin{tabular}{|r|r|l|l|l|r|r|r|}
\hline \multicolumn{7}{|c|}{ Conjugacy class representatives for $G_{4}$} \\
\hline $\operatorname{Order}(g)$ & 1 & 4 & 3 & 6 & 6 & 3 & 2 \\
\hline $\operatorname{det}(g)$ & 1 & 1 & $\omega$ & $\omega$ & $\omega^{-1}$ & $\omega^{-1}$ & 1 \\
\hline$|\mathcal{C}(g)|$ & 1 & 6 & 4 & 4 & 4 & 4 & 1 \\
\hline$\left|Z_{G}(g)\right|$ & 24 & 4 & 6 & 6 & 6 & 6 & 24 \\
\hline
\end{tabular}

The elements with determinant 1 and order more than 2 in $G_{4}$ all have order 4 . If $g$ is an element of order 4 , then $\left|Z_{G}(g)\right|=4$ and every element of $Z_{G}(g)$ has determinant 1 since $Z_{G}(g)$ is generated by $g$. Hence, by Theorem $1.9 \mathrm{~b}$ and Lemma 2.2, $a_{g}$ can be nonzero for a graded Hecke algebra for $G_{4}$ exactly when $g$ has order 
4. Thus, the dimension of the space of parameters for graded Hecke algebras of $G_{4}$ is 1 .

The rank 2 group $G_{12}$ has order 48 . The computer software GAP provides the following information about the conjugacy classes of $G_{12}$.

\begin{tabular}{|r|r|r|r|r|r|r|r|l|}
\hline \multicolumn{10}{|c|}{ Conjugacy class representatives for $G_{12}$} \\
\hline $\operatorname{Order}(g)$ & 1 & 2 & 8 & 6 & 8 & 2 & 3 & 4 \\
\hline $\operatorname{det}(g)$ & 1 & -1 & -1 & 1 & -1 & 1 & 1 & 1 \\
\hline$|\mathcal{C}(g)|$ & 1 & 12 & 6 & 8 & 6 & 1 & 8 & 6 \\
\hline$\left|Z_{G}(g)\right|$ & 48 & 4 & 8 & 6 & 8 & 48 & 6 & 8 \\
\hline
\end{tabular}

If $g$ is an element in $G_{12}$ with order more than 2 and determinant 1, then $g$ has order 3,4 , or 6 . Let $h$ be any element of order 8 . Then $h$ has determinant -1 and commutes with $h^{2}$ of order 4 . Hence, by Theorem $1.9 \mathrm{~b}$, if $g$ has order 4 , then $a_{g}=0$. Let $g_{6}$ be a representative from the class of elements of order 6 . Since $\left|Z_{G}\left(g_{6}\right)\right|=6, Z_{G}\left(g_{6}\right)$ is generated by $g_{6}$ and hence every element of $Z_{G}\left(g_{6}\right)$ has determinant 1 . We can choose $g_{6}^{2}$ as a representative for the conjugacy class of elements of order 3 . As $g_{6}$ and $g_{6}^{2}$ commute, $\left\langle g_{6}\right\rangle \subset Z_{G}\left(g_{6}^{2}\right)$. But $\left|\left\langle g_{6}\right\rangle\right|=$ $6=\left|Z_{G}\left(g_{3}\right)\right|$, so $Z_{G}\left(g_{6}^{2}\right)$ is generated by $g_{6}$ and every element of $Z_{G}\left(g_{3}\right)$ has determinant 1 . Thus, $a_{g}$ can be nonzero for a graded Hecke algebra $A$ for $G_{12}$ exactly when $g$ has order 3 or 6 . Thus, the dimension of the space of parameters of graded Hecke algebras for $G_{12}$ is 2 .

The rank 3 group $G_{24}$ has order 336 . Note that $-1 \in G_{24}$ since $Z(G)=\{ \pm 1\}$. Up to $G$-orbits, there are two codimension 2 subspaces, $L$ and $M$, that are equal to $V^{g}$ for some $g \in G_{24}$ (see [OT, App. C, Table C.5]). Furthermore, $\operatorname{Stab}(L) \cong A_{2}$ and $\operatorname{Stab}(M) \cong B_{2}$. We need only consider elements of order 3 in $\operatorname{Stab}(L) \cong A_{2}$ and of order 4 in $\operatorname{Stab}(M) \cong B_{2}$ (as the rest have order 1 or 2 ). In $G_{24}$, there is only one conjugacy class of elements of order 3 and only one conjugacy class of elements of order 4 and determinant 1. The table below (obtained using GAP) records information about these classes.

\begin{tabular}{|c|c|c|}
\hline \multicolumn{3}{|c|}{ Certain classes of $G_{24}$} \\
\hline $\operatorname{Order}(g)$ & 3 & 4 \\
\hline $\operatorname{det}(g)$ & 1 & 1 \\
\hline$|\mathcal{C}(g)|$ & 56 & 42 \\
\hline$\left|Z_{G}(g)\right|$ & 6 & 8 \\
\hline
\end{tabular}

If $g$ has order $3, Z_{G}(g)$ must contain $1, g, g^{2}$, and -1 , and hence $Z_{G}(g)$ is generated by these elements since $\left|Z_{G}(g)\right|=6$. Thus all elements of $Z_{G}(g)$ have determinant 1 
on $\left(V^{g}\right)^{\perp}$. If $g$ has order 4 and determinant 1 , then $Z_{G}(g)$ must contain $1, g, g^{2}, g^{3}$, and -1 , elements which all have determinant 1 on $\left(V^{g}\right)^{\perp}$. Since $\left|Z_{G}(g)\right|=8$, these elements generate $Z_{G}(g)$ and so every element of $Z_{G}(g)$ has determinant 1 on $\left(V^{g}\right)^{\perp}$. Hence, $a_{g}$ can be nonzero for a graded Hecke algebra of $G_{24}$ exactly when $g$ has order 3 or $g$ has order 4 and determinant 1 . Thus, the dimension of the space of parameters for graded Hecke algebras for $G_{24}$ is 2 .

The group $G_{33}$ is the only exceptional complex reflection group of rank 5 . It has order $72 \cdot 6$ ! and degrees $4,6,10,12,18$. There are 45 reflecting hyperplanes and the corresponding reflections all have order 2 . Up to $G$-orbits, there are two codimension 2 subspaces, $L$ and $M$, that are equal to $V^{g}$ for some $g \in G_{33}$ (see [OT, App. C, Table C.14]). Furthermore, $\operatorname{Stab}(L) \cong A_{1} \times A_{1}$ and $\operatorname{Stab}(M) \cong A_{2}$. We need not consider the case where $V^{g}=L$ since then $g$ has order 2 and hence $a_{g}=0$ for any graded Hecke algebra by Proposition $2.2 \mathrm{~b}$.

We use a presentation for $G_{33}$ in six coordinates from [ST]: Let $V=\mathbb{C}^{6}$ with standard coordinate functions $x_{i}$ and consider the group generated by order 2 reflections about the hyperplanes $H_{1}=\left\{x_{2}-x_{3}=0\right\}, H_{2}=\left\{x_{3}-x_{4}=0\right\}$, $H_{3}=\left\{x_{1}-x_{2}=0\right\}, H_{4}=\left\{x_{1}-\omega x_{2}=0\right\}, H_{5}=\left\{x_{1}+x_{2}+x_{3}+x_{4}+x_{5}+x_{6}=0\right\}$, where $\omega$ is a primitive cube root of unity. The fixed point space of this (reducible) group is $Y=H_{1} \cap \cdots \cap H_{5}=\{(0,0,0,0, x,-x) \mid x \in \mathbb{C}\}$, and $G_{33}$ is just the restriction to $Y^{\perp}$. Let $s_{i}$ be the order 2 reflection about $H_{i}$. Let $g=s_{1} s_{3}$. Then $V^{g}=H_{1} \cap H_{3}$ and $\operatorname{Stab}\left(V^{g}\right) \cong A_{2}$. Let $h=\left(s_{1} s_{3} s_{4}\right)^{2}$, the diagonal matrix with diagonal $\{\omega, \omega, \omega, 1,1,1\}$. Then $h$ acts as $\omega$ times the identity on $\left(V^{g}\right)^{\perp}$ as $\left(V^{g}\right)^{\perp} \subseteq \mathbb{C}$-span $\left\{x_{1}, x_{2}, x_{3}\right\}$. Hence, $h$ commutes with $g$. But $\left(V^{g}\right)^{\perp}$ has dimension 2 and $h$ has determinant $\omega^{2} \neq 1$ on $\left(V^{g}\right)^{\perp}$. Thus, by Theorem $1.9 \mathrm{~b}$ and Lemma 2.2, $a_{g}=0$ for any graded Hecke algebra. The same argument applied to $Y^{\perp}$ shows that $G_{33}$ has no nontrivial graded Hecke algebras. In summary, the dimension of the space of parameters for graded Hecke algebras for $G_{33}$ is zero.

\begin{tabular}{|l|c|}
\hline \multicolumn{1}{|c|}{ Group } & $g$ with $a_{g} \neq 0$ \\
\hline$G_{4}$ & $\operatorname{Order}(g)=4$ \\
$G_{12}$ & $\operatorname{Order}\left(g_{1}\right)=3$ and $\operatorname{Order}\left(g_{2}\right)=6$ \\
$G_{24}$ & $\operatorname{Order}\left(g_{1}\right)=3$ and $\operatorname{Order}\left(g_{2}\right)=4, \operatorname{det}\left(g_{2}\right)=1$ \\
\hline
\end{tabular}

Table 3. Graded Hecke algebras for exceptional complex reflection groups

\section{The graded Hecke algebras $H_{\mathrm{gr}}$}

In $[\mathrm{Lu}]$, Lusztig gives a definition of graded Hecke algebras for real reflection groups which is different from the definition in Section 1, which applies to more general groups. It is not obvious that Lusztig's algebras are examples of the graded Hecke 
algebras defined in Section 1. In this section, we show explicitly how the definition of Section 1 includes Lusztig's algebras.

Let $W$ be a finite real reflection group acting on $V$ and let $R$ be the root system of $W$. Let $\alpha_{1}, \ldots, \alpha_{n}$ be a choice of simple roots in $R$ and let $s_{1}, \ldots, s_{n}$ be the corresponding simple reflections in $W$. Let $s_{\alpha}$ be the reflection in the root $\alpha$ so that, for $v \in V$,

$$
s_{\alpha} v=v-\left\langle v, \alpha^{\vee}\right\rangle \alpha, \quad \text { where } \alpha^{\vee}=2 \alpha /\langle\alpha, \alpha\rangle \text {. }
$$

Let $R^{+}=\{\alpha>0\}$ denote the set of positive roots in $R$.

Let $k_{\alpha}$ be fixed complex numbers indexed by the roots $\alpha \in R$ satisfying

$$
k_{w \alpha}=k_{\alpha}, \quad \text { for all } w \in W, \alpha \in R \text {. }
$$

This amounts to a choice of either one or two "parameters", depending on whether all roots in $R$ are the same length or not. As in Section 1, let $\mathbb{C} W=\mathbb{C}$-span $\left\{t_{g} \mid g \in\right.$ $W\}$, with $t_{g} t_{h}=t_{g h}$, and let $S(V)$ be the symmetric algebra of $V$. Lusztig [Lu] defines the "graded Hecke algebra" with parameters $\left\{k_{\alpha}\right\}$ to be the unique algebra structure $H_{\text {gr }}$ on the vector space $S(V) \otimes \mathbb{C} W$ such that

$$
\begin{aligned}
S(V) & =S(V) \otimes 1 \text { is a subalgebra of } H_{\mathrm{gr}}, \\
\mathbb{C} W & =1 \otimes \mathbb{C} W \text { is a subalgebra of } H_{\mathrm{gr}},
\end{aligned}
$$

and

$$
\begin{aligned}
t_{s_{i}} v= & \left(s_{i} v\right) t_{s_{i}}-k_{\alpha_{i}}\left\langle v, \alpha_{i}^{\vee}\right\rangle, \\
& \text { for all } v \in V \text { and simple reflections } s_{i} \text { in the simple roots } \alpha_{i} .
\end{aligned}
$$

We shall show that every algebra $H_{\text {gr }}$ as defined by $(3.2 \mathrm{a}-\mathrm{c})$ is a graded Hecke algebra $A$ for a specific set of skew symmetric bilinear forms $a_{g}$.

Let $k_{\alpha} \in \mathbb{C}$ as in (3.1). Use the notation

$$
h=\frac{1}{2} \sum_{\alpha>0} k_{\alpha} \alpha^{\vee} t_{s_{\alpha}}, \quad \text { so that } \quad\langle v, h\rangle=\frac{1}{2} \sum_{\alpha>0} k_{\alpha}\left\langle v, \alpha^{\vee}\right\rangle t_{s_{\alpha}}
$$

for $v \in V$. The element $h$ should be viewed as an element of $V \otimes \mathbb{C} W$, and $\langle v, h\rangle \in \mathbb{C} W$. With this notation, let $A$ be the algebra (as in Section 1) generated by $V$ and $\mathbb{C} W$ with relations

$$
t_{g} v=(g v) t_{g} \quad \text { and } \quad[v, w]=-[\langle v, h\rangle,\langle w, h\rangle], \quad \text { for } v, w \in V, g \in W \text {. }
$$


Note that $A$ is defined by the bilinear forms

$$
a_{g}(v, w)=\frac{1}{4} \sum_{\substack{\alpha, \beta>0 \\ g=s_{\alpha} s_{\beta}}} k_{\alpha} k_{\beta}\left(\left\langle v, \beta^{\vee}\right\rangle\left\langle w, \alpha^{\vee}\right\rangle-\left\langle v, \alpha^{\vee}\right\rangle\left\langle w, \beta^{\vee}\right\rangle\right) .
$$

The following theorem shows that the algebra $A$ satisfies the defining conditions $(3.2 \mathrm{a}-\mathrm{c})$ of the algebra $H_{\mathrm{gr}}$.

Theorem 3.5. Let $W$ be a finite real reflection group and let $A$ be the algebra defined by (3.4).

(a) As vector spaces, $A \cong S(V) \otimes \mathbb{C W}$ (and hence, $A$ is a graded Hecke algebra).

(b) If $\tilde{v}=v-\langle v, h\rangle$ for $v \in V$, then

$$
[\tilde{v}, \tilde{w}]=0 \quad \text { and } \quad t_{s_{i}} \tilde{v}=\left(\widetilde{s_{i} v}\right) t_{s_{i}}-k_{\alpha_{i}}\left\langle v, \alpha_{i}^{\vee}\right\rangle
$$

for all $v, w \in V$ and simple reflections $s_{i}$ in $W$.

Proof. First note that if $u, v \in V$ then

$$
[u,\langle v, h\rangle]=\frac{1}{2} \sum_{\alpha>0} k_{\alpha}\left\langle v, \alpha^{\vee}\right\rangle\left\langle u, \alpha^{\vee}\right\rangle \alpha t_{s_{\alpha}}=[v,\langle u, h\rangle]
$$

Thus, for $u, v, w \in V$,

$$
\begin{aligned}
{[u,[v, w]]+} & {[w,[u, v]]+[v,[w, u]] } \\
& =[u,[\langle w, h\rangle,\langle v, h\rangle]]+[w,[\langle v, h\rangle,\langle u, h\rangle]]+[v,[\langle u, h\rangle,\langle w, h\rangle]] \\
& =[[u,\langle w, h\rangle],\langle v, h\rangle]+[\langle w, h\rangle,[u,\langle v, h\rangle]]+[[w,\langle v, h\rangle],\langle u, h\rangle] \\
& \quad+[\langle v, h\rangle,[w,\langle u, h\rangle]]+[[v,\langle u, h\rangle],\langle w, h\rangle]+[\langle u, h\rangle],[v,\langle w, h\rangle]] \\
& =[[w,\langle u, h\rangle],\langle v, h\rangle]+[\langle w, h\rangle,[v,\langle u, h\rangle]]+[[v,\langle w, h\rangle],\langle u, h\rangle] \\
& \quad+[\langle v, h\rangle,[w,\langle u, h\rangle]]+[[v,\langle u, h\rangle],\langle w, h\rangle]+[\langle u, h\rangle,[v,\langle w, h\rangle]] \\
& =0 .
\end{aligned}
$$

For $v \in V, h \in W$, and $s_{i}$ a simple reflection,

$$
\begin{aligned}
t_{s_{i}}\langle v, h\rangle t_{s_{i}} & =\frac{1}{2} \sum_{\alpha>0} k_{\alpha}\left\langle v, \alpha^{\vee}\right\rangle t_{s_{s_{i}}}=\left(\frac{1}{2} \sum_{\alpha>0} k_{\alpha}\left\langle v, s_{i} \alpha^{\vee}\right\rangle t_{s_{\alpha}}\right)+k_{\alpha_{i}}\left\langle v, \alpha_{i}^{\vee}\right\rangle t_{s_{i}} \\
& =\left(\frac{1}{2} \sum_{\alpha>0} k_{\alpha}\left\langle s_{i} v, \alpha^{\vee}\right\rangle t_{s_{\alpha}}\right)+k_{\alpha_{i}}\left\langle v, \alpha_{i}^{\vee}\right\rangle t_{s_{i}}=\left\langle s_{i} v, h\right\rangle+k_{\alpha_{i}}\left\langle v, \alpha_{i}^{\vee}\right\rangle t_{s_{i}} .
\end{aligned}
$$


Using this equality, we obtain

$$
\begin{aligned}
t_{s_{i}}[v, w] t_{s_{i}}= & -t_{s_{i}}[\langle v, h\rangle,\langle w, h\rangle] t_{s_{i}} \\
= & -\left[\left\langle s_{i} v, h\right\rangle+k_{\alpha_{i}}\left\langle v, \alpha_{i}^{\vee}\right\rangle t_{s_{i}},\left\langle s_{i} w, h\right\rangle+k_{\alpha_{i}}\left\langle w, \alpha_{i}^{\vee}\right\rangle t_{s_{i}}\right] \\
= & {\left[s_{i} v, s_{i} w\right]-k_{\alpha_{i}}\left\langle v, \alpha_{i}^{\vee}\right\rangle\left[t_{s_{i}},\left\langle s_{i} w, h\right\rangle\right]-k_{\alpha_{i}}\left\langle w, \alpha_{i}^{\vee}\right\rangle\left[\left\langle s_{i} v, h\right\rangle, t_{s_{i}}\right] } \\
= & {\left[s_{i} v, s_{i} w\right]-k_{\alpha_{i}}\left\langle v, \alpha_{i}^{\vee}\right\rangle\left(t_{s_{i}}\left\langle s_{i} w, h\right\rangle t_{s_{i}}-\left\langle s_{i} w, h\right\rangle\right) t_{s_{i}} } \\
& \quad+k_{\alpha_{i}}\left\langle w, \alpha_{i}^{\vee}\right\rangle\left(t_{s_{i}}\left\langle s_{i} v, h\right\rangle t_{s_{i}}-\left\langle s_{i} v, h\right\rangle\right) t_{s_{i}} \\
= & {\left[s_{i} v, s_{i} w\right]-k_{\alpha_{i}}\left\langle v, \alpha_{i}^{\vee}\right\rangle\left(\langle w, h\rangle t_{s_{i}}+k_{\alpha_{i}}\left\langle s_{i} w, \alpha_{i}^{\vee}\right\rangle-\left\langle s_{i} w, h\right\rangle t_{s_{i}}\right) } \\
& \quad+k_{\alpha_{i}}\left\langle w, \alpha_{i}^{\vee}\right\rangle\left(\langle v, h\rangle t_{s_{i}}+k_{\alpha_{i}}\left\langle s_{i} v, \alpha_{i}^{\vee}\right\rangle-\left\langle s_{i} v, h\right\rangle t_{s_{i}}\right) \\
= & {\left[s_{i} v, s_{i} w\right]-k_{\alpha_{i}}\left\langle v, \alpha_{i}^{\vee}\right\rangle\left\langle w, \alpha_{i}^{\vee}\right\rangle\left\langle\alpha_{i}, h\right\rangle t_{s_{i}}-k_{\alpha_{i}}^{2}\left\langle v, \alpha_{i}^{\vee}\right\rangle\left\langle w, s_{i} \alpha_{i}^{\vee}\right\rangle } \\
& \quad+k_{\alpha_{i}}\left\langle w, \alpha_{i}^{\vee}\right\rangle\left\langle v, \alpha_{i}^{\vee}\right\rangle\left\langle\alpha_{i}, h\right\rangle t_{s_{i}}+k_{\alpha_{i}}^{2}\left\langle w, \alpha_{i}^{\vee}\right\rangle\left\langle v, s_{i} \alpha_{i}^{\vee}\right\rangle \\
= & {\left[s_{i} v, s_{i} w\right] . }
\end{aligned}
$$

The two identities (3.6) and (3.8), as in (1.3) and (1.4), show that the algebra $A$ is isomorphic to $S(V) \otimes \mathbb{C} W$.

(b) This can now be proved by direct computation. If $v, w \in V$ then

$[\tilde{v}, \tilde{w}]=[v-\langle v, h\rangle, w-\langle w, h\rangle]=[v, w]+[\langle v, h\rangle,\langle w, h\rangle]-[v,\langle w, h\rangle]+[w,\langle v, h\rangle]=0$

by equation (3.4) and equation $(*)$ in the proof of Theorem 3.5. If $v \in V$ and $s_{i}$ is a simple reflection then, by (3.7),

$t_{s_{i}} \tilde{v} t_{s_{i}}=t_{s_{i}} v t_{s_{i}}-t_{s_{i}}\langle v, h\rangle t_{s_{i}}=s_{i} v-\left\langle s_{i} v, h\right\rangle-k_{\alpha_{i}}\left\langle v, \alpha_{i}^{\vee}\right\rangle t_{s_{i}}=\widetilde{s_{i} v}-k_{\alpha_{i}}\left\langle v, \alpha_{i}^{\vee}\right\rangle t_{s_{i}}$.

Theorem 3.5b shows that if $A$ is the graded Hecke algebra defined by (3.4), then the elements $\tilde{v}$, for $v \in V$, generate a subalgebra of $A$ isomorphic to $S(V)$ and these elements together with the $t_{s_{i}}$ satisfy the relations of (3.2c). Since part (a) of Theorem 3.5 shows that $A$ is isomorphic to $S(V) \otimes \mathbb{C} W$ as a vector space, it follows that $A$ satisfies the conditions $(3.2 \mathrm{a}-\mathrm{c})$, relations which uniquely define the graded Hecke algebra $A$. Thus, Lusztig's algebras are special cases of the graded Hecke algebras defined in Section 1. Furthermore, by comparing the dimensions of the parameter spaces, we see that there are graded Hecke algebras that are not isomorphic to algebras defined by Lusztig for the Coxeter groups $F_{4}, H_{3}, H_{4}$, and $I_{2}(m)$. 


\section{Examples}

\section{A. The symmetric group $G(1,1, n)=S_{n}$}

Let $V$ be an $n$ dimensional vector space with orthonormal basis $v_{1}, \ldots, v_{n}$ and let $S_{n}$ act on $V$ by permuting the $v_{i}$. Let $A$ be a graded Hecke algebra for $S_{n}$. Any element which is a product of two reflections is conjugate to $(1,2,3)$ or $(1,2)(3,4)$. The element $(1,2)(3,4)$ has order 2 and so, in the algebra $A$,

$$
\left[v_{i}, v_{j}\right]=\sum_{k \neq i, j}\left(a_{(i, j, k)}\left(v_{i}, v_{j}\right) t_{(i, j, k)}+a_{(j, i, k)}\left(v_{i}, v_{j}\right) t_{(j, i, k)}\right),
$$

since $v_{i}$ or $v_{j}$ is in $V^{g}=\operatorname{ker} a_{g}$ for all other three cycles $g$. Since, by (1.6), $a_{(j, i, k)}\left(v_{i}, v_{j}\right)=a_{(i, j, k)}\left(v_{j}, v_{i}\right)=-a_{(i, j, k)}\left(v_{i}, v_{j}\right)$, the graded Hecke algebra $A$ is defined by the relations

$$
\left[v_{i}, v_{j}\right]=\beta \sum_{k \neq i, j}\left(t_{(i, j, k)}-t_{(j, i, k)}\right) \quad \text { and } \quad t_{w} v_{i}=v_{w(i)} t_{w}
$$

where $w \in S_{n}, 1 \leq i, j \leq n, i \neq j$, and $\beta=a_{(1,2,3)}\left(v_{1}, v_{2}\right)$.

Let $k \in \mathbb{C}$. Then, with $h$ as in (3.3),

$$
\begin{aligned}
\left\langle v_{i}, h\right\rangle & =\frac{1}{2} \sum_{\ell<m} k\left\langle v_{i}, v_{\ell}-v_{m}\right\rangle t_{(\ell, m)} \\
& =\frac{k}{2}\left(\sum_{i<\ell} t_{(i, \ell)}-\sum_{i>\ell} t_{(\ell, i)}\right)=\frac{k}{2} \sum_{i \neq \ell} \operatorname{sgn}(\ell-i) t_{(i, \ell)} .
\end{aligned}
$$

If $f \in \mathbb{C} S_{n}$, let $\left.f\right|_{t_{g}}$ denote the coefficient of $t_{g}$ in $f$. Let $A$ be the graded Hecke algebra defined by the relations in (4.1) with

$$
\begin{aligned}
\beta & =a_{(i, j, \ell)}\left(v_{i}, v_{j}\right)=\left.\left[\left\langle v_{i}, h\right\rangle,\left\langle v_{j}, h\right\rangle\right]\right|_{t_{(i, j, \ell)}} \\
& =\left.\left(k^{2} / 4\right)\left(t_{(i, \ell)} t_{(j, \ell)}+t_{(i, j)} t_{(i, \ell)}-t_{(j, \ell)} t_{(i, j)}\right)\right|_{t_{(i, j, \ell)}}=k^{2} / 4 .
\end{aligned}
$$

If $\tilde{v}_{i}=v_{i}-\left\langle v_{i}, h\right\rangle$ and $s_{i}$ is the simple reflection $(i, i+1)$ then, by Theorem 3.5,

$$
\begin{aligned}
\tilde{v}_{i} \tilde{v}_{j} & =\tilde{v}_{j} \tilde{v}_{i}, \quad t_{s_{i}} \tilde{v}_{i}=\tilde{v}_{i+1} t_{s_{i}}+k, \quad t_{s_{i}} \tilde{v}_{i+1}=\tilde{v}_{i} t_{s_{i}}-k, \quad \text { and } \\
t_{s_{j}} \tilde{v}_{i} & =\tilde{v}_{i} t_{s_{j}}, \quad \text { for }|i-j|>1,
\end{aligned}
$$

and the algebra $A$ is the graded Hecke algebra $H_{\text {gr }}$ for $S_{n}$ which is defined in Section 3 . When $k=1$, the map

$$
\begin{array}{rlc}
A & \longrightarrow & \mathbb{C} S_{n} \\
t_{w} & \longmapsto & t_{w} \\
v_{i} & \longmapsto & \frac{1}{2} \sum_{\ell \neq i} t_{(i, \ell)}
\end{array}
$$

is a surjective algebra homomorphism. 
4B. The hyperoctahedral group $G(2,1, n)=W B_{n}$

We use the notation from Section $2 \mathrm{~B}$ so that the group $G(2,1, n)$ is acting by orthogonal matrices on the $n$ dimensional vector space $V$ with orthonormal basis $\left\{v_{1}, \ldots, v_{n}\right\}$. In this case, $\xi_{i}$ denotes the diagonal matrix with all ones on the diagonal except for -1 in the $(i, i)$ th entry.

Let $A$ be a graded Hecke algebra for $G(2,1, n)$. If $\beta_{1}=a_{(i, j, k)}\left(v_{i}, v_{j}\right)$ and $\beta_{2}=a_{\xi_{1}(1,2)}\left(v_{1}, v_{2}\right)$, then, in the algebra $A$,

$$
\begin{aligned}
{\left[v_{i}, v_{j}\right]=} & \beta_{2}\left(t_{\xi_{1}(1,2)}-t_{\xi_{2}(1,2)}\right) \\
& +\beta_{1} \sum_{\ell \neq i, j}\left(\begin{array}{c}
t_{(i, j, \ell)}-t_{\xi_{i} \xi_{\ell}(i, j, \ell)}-t_{\xi_{i} \xi_{j}(i, j, \ell)}+t_{\xi_{j} \xi_{\ell}(i, j, \ell)} \\
+t_{\xi_{i} \xi_{j}(j, i, \ell)}+t_{\xi_{j} \xi_{\ell}(j, i, \ell)}-t_{\xi_{i} \xi_{\ell}(j, i, \ell)}-t_{(j, i, \ell)}
\end{array}\right) .
\end{aligned}
$$

Let $k_{s}, k_{\ell} \in \mathbb{C}$. Then, with $h$ as in (3.3),

$$
\begin{aligned}
\left\langle v_{i}, h\right\rangle & =\frac{k_{s}}{2} \sum_{\ell}\left\langle v_{i}, 2 v_{\ell}\right\rangle t_{\xi_{\ell}}-\frac{k_{\ell}}{2} \sum_{\ell<m}\left\langle v_{i}, v_{\ell}-v_{m}\right\rangle t_{(\ell, m)}-\frac{k_{\ell}}{2} \sum_{\ell<m}\left\langle v_{i}, v_{\ell}+v_{m}\right\rangle t_{\xi_{\ell}(\ell, m)} \\
& =k_{s} t_{\xi_{i}}-\frac{k_{\ell}}{2}\left(\sum_{i<\ell}\left(t_{(i, \ell)}+t_{\xi_{i} \xi_{\ell}(i, \ell)}\right)+\sum_{i>\ell}\left(-t_{(i, \ell)}+t_{\xi_{i} \xi_{\ell}(i, \ell)}\right)\right) .
\end{aligned}
$$

If $f \in \mathbb{C} G(2,1, n)$, let $\left.f\right|_{t_{g}}$ denote the coefficient of $t_{g}$ in $f$. With notation as in (4.6), let $A$ be the graded Hecke algebra for $G(2,1, n)$ with

$$
\begin{aligned}
\beta_{1} & =a_{(i, j, \ell)}\left(v_{i}, v_{j}\right)=\left.\left[\left\langle v_{i}, h\right\rangle,\left\langle v_{j}, h\right\rangle\right]\right|_{t_{(i, j, \ell)}} \\
& =\left.\left(k_{\ell}^{2} / 4\right)\left(t_{(i, \ell)} t_{(j, \ell)}+t_{(i, j)} t_{(i, \ell)}-t_{(j, \ell)} t_{(i, j)}\right)\right|_{t_{(i, j, \ell)}}=k_{\ell}^{2} / 4, \quad \text { and } \\
\beta_{2} & =\left.\left[\left\langle v_{i}, h\right\rangle,\left\langle v_{j}, h\right\rangle\right]\right|_{t_{\xi_{i}(i, j)}} \\
& =\left.(1 / 2) k_{s} k_{\ell}\left(-t_{\xi_{i}} t_{(i, j)}+t_{(i, j)} t_{\xi_{j}}-t_{\xi_{j}} t_{\xi_{i} \xi_{j}(i, j)}-t_{\xi_{i} \xi_{j}(i, j)} t_{\xi_{i}}\right)\right|_{t_{\xi_{i}(i, j)}}=-k_{s} k_{\ell} .
\end{aligned}
$$

If $\tilde{v}_{i}=v_{i}-\left\langle v_{i}, h\right\rangle$, then, by Theorem 3.5, the $\tilde{v}_{i}$ commute and the algebra $A$ is the algebra $H_{\mathrm{gr}}$ for $W B_{n}$ defined in Section 3.

\section{C. The type $D_{n}$ Weyl group $G(2,2, n)=W D_{n}$}

We shall use the notation from Section 2B so that the group $G(2,2, n)$ is acting by orthogonal matrices on the $n$ dimensional vector space $V$ with orthonormal basis $\left\{v_{1}, \ldots, v_{n}\right\}$. This is an index 2 subgroup of $G(2,1, n)$, and our notation is the same as used above for $W B_{n}$.

Let $A$ be a graded Hecke algebra for $G(2,2, n)$. If $\beta=a_{(i, j, k)}\left(v_{i}, v_{j}\right)$ then, in the algebra $A$,

$$
\left[v_{i}, v_{j}\right]=\beta \sum_{\ell \neq i, j}\left(\begin{array}{c}
t_{(i, j, k)}-t_{\xi_{i} \xi_{\ell}(i, j, \ell)}-t_{\xi_{i} \xi_{j}(i, j, \ell)}+t_{\xi_{j} \xi_{\ell}(i, j, \ell)} \\
+t_{\xi_{i} \xi_{j}(j, i, \ell)}+t_{\xi_{j} \xi_{\ell}(j, i, \ell)}-t_{\xi_{i} \xi_{\ell}(j, i, \ell)}-t_{(j, i, \ell)}
\end{array}\right) .
$$


Let $k \in \mathbb{C}$. Then, with $h$ as in $(3.3)$,

$$
\left\langle v_{i}, h\right\rangle=\frac{k}{2}\left(\sum_{i<\ell}\left(t_{(i, \ell)}+t_{\xi_{i} \xi_{\ell}(i, \ell)}\right)+\sum_{i>\ell}\left(-t_{(i, \ell)}+t_{\xi_{i} \xi_{\ell}(i, \ell)}\right)\right) .
$$

If $f \in \mathbb{C} G(2,2, n)$, let $\left.f\right|_{t_{g}}$ denote the coefficient of $t_{g}$ in $f$. With notation as in (4.8), let $A$ be the graded Hecke algebra for $G(2,2, n)$ with

$$
\begin{aligned}
\beta & =a_{(i, j, \ell)}\left(v_{i}, v_{j}\right)=\left.\left[\left\langle v_{i}, h\right\rangle,\left\langle v_{j}, h\right\rangle\right]\right|_{t_{(i, j, \ell)}} \\
& =\left.\left(k^{2} / 4\right)\left(t_{(i, \ell)} t_{(j, \ell)}+t_{(i, j)} t_{(i, \ell)}-t_{(j, \ell)} t_{(i, j)}\right)\right|_{t_{(i, j, \ell)}}=k^{2} / 4 .
\end{aligned}
$$

If $\tilde{v}_{i}=v_{i}-\left\langle v_{i}, h\right\rangle$, then, by Theorem 3.5, the $\tilde{v}_{i}$ commute and the algebra $A$ is the algebra $H_{\text {gr }}$ for $W D_{n}$ defined in Section 3.

\section{D. The dihedral group $I_{2}(r)=G(r, r, 2)$ of order $2 r$}

We shall use the notation for $G(r, r, 2)$ from Section 2B so that the group $G(r, r, 2)$ is acting by unitary matrices on the 2 dimensional vector space $V$ with orthonormal basis $\left\{v_{1}, v_{2}\right\}$. The group $G(r, r, 2)$ is realized as a real reflection group by using the basis

$$
\varepsilon_{1}=\frac{1}{\sqrt{2}}\left(v_{1}+v_{2}\right), \quad \varepsilon_{2}=\frac{-1}{i \sqrt{2}}\left(v_{1}-v_{2}\right) .
$$

This basis is also orthonormal and, with respect to this basis, $G(r, r, 2)$ acts by the matrices

$$
\left(\begin{array}{cc}
\cos (2 \pi m / r) & \mp \sin (2 \pi m / r) \\
\sin (2 \pi m / r) & \pm \cos (2 \pi m / r)
\end{array}\right), \quad 0 \leq m \leq r-1
$$

Let $A$ be a graded Hecke algebra for $G(r, r, 2)$. The conjugacy classes of elements which are products of two reflections are $\left\{\xi_{1}^{k} \xi_{2}^{-k}, \xi_{1}^{-k} \xi_{2}^{k}\right\}, 0<k<r / 2$. Then, in the algebra $A$,

$$
\left[\varepsilon_{1}, \varepsilon_{2}\right]=\sum_{0<k<r / 2} \beta_{k}\left(t_{\xi_{1}^{k} \xi_{2}^{-k}}-t_{\xi_{1}^{-k} \xi_{2}^{k}}\right), \quad \text { where } \quad \beta_{k}=a_{\xi_{1}^{k} \xi_{2}^{-k}}\left(\varepsilon_{1}, \varepsilon_{2}\right)
$$

When $r$ is even, there are two conjugacy classes of reflections

$$
\left\{\xi_{1}^{2 k} \xi_{2}^{-2 k}(1,2) \mid 0 \leq k<r / 2\right\} \quad \text { and } \quad\left\{\xi_{1}^{2 k+1} \xi_{2}^{-(2 k+1)}(1,2) \mid 0 \leq k<r / 2\right\}
$$

The reflection $\xi_{1}^{m} \xi_{2}^{-m}(12)$ is the reflection in the line perpendicular to the vector

$$
\alpha_{m}=\sin (-2 \pi m / 2 r) \varepsilon_{1}+\cos (-2 \pi m / 2 r) \varepsilon_{2},
$$


and the vectors $\alpha_{m}$ can be taken as a root system for $G(r, r, 2)$. With $h$ as in (3.3) and $k_{s}, k_{\ell} \in \mathbb{C}$,

$$
\begin{aligned}
\left\langle\varepsilon_{1}, h\right\rangle= & \sum_{0 \leq k<r / 2}\left(k_{s} \sin (-2 k 2 \pi / 2 r) t_{\xi_{1}^{2 k} \xi_{2}^{-2 k}(1,2)}\right. \\
& \left.+k_{\ell} \sin (-(2 k+1) 2 \pi / 2 r) t_{\xi_{1}^{2 k+1} \xi_{2}^{-(2 k+1)}(1,2)}\right), \\
\left\langle\varepsilon_{2}, h\right\rangle= & \sum_{0 \leq k<r / 2}\left(k_{s} \cos (-2 k 2 \pi / 2 r) t_{\xi_{1}^{2 k} \xi_{2}^{-2 k}(1,2)}\right. \\
& \left.+k_{\ell} \cos (-(2 k+1) 2 \pi / 2 r) t_{\xi_{1}^{2 k+1} \xi_{2}^{-(2 k+1)}(1,2)}\right) .
\end{aligned}
$$

If $f \in \mathbb{C} G(r, r, 2)$, let $\left.f\right|_{t_{g}}$ denote the coefficient of $t_{g}$ in $f$. With notation as in 4.10 , let $A$ be the graded Hecke algebra for $G(r, r, 2)$ with

$$
\begin{aligned}
& \beta_{k}= a_{\xi_{1}^{k} \xi_{2}^{-k}}\left(\varepsilon_{1}, \varepsilon_{2}\right)=\left.\left[\left\langle\varepsilon_{1}, h\right\rangle,\left\langle\varepsilon_{2}, h\right\rangle\right]\right|_{t_{\xi_{1}^{k} \xi_{2}^{-k}}} \\
&= \begin{cases}\sin (k 2 \pi / 2 r) r k_{s} k_{\ell} & \text { if } k \text { is odd } \\
\sin (k 2 \pi / 2 r) \frac{r}{2}\left(k_{s}^{2}+k_{\ell}^{2}\right) & \text { if } k \text { is even. }\end{cases}
\end{aligned}
$$

If $\tilde{\varepsilon}_{i}=\varepsilon_{i}-\left\langle\varepsilon_{i}, h\right\rangle$, then by Theorem 3.5, the $\tilde{\varepsilon}_{i}$ commute and the algebra $A$ is the algebra $H_{\text {gr }}$ for $I_{2}(r)$ defined in Section 3 .

When $r$ is odd, all aspects of the calculation in (4.11) and (4.12) are the same as for the case $r$ even except that there is only one conjugacy class of reflections, $\left\{\xi_{1}^{k} \xi_{2}^{-k}(1,2) \mid 0 \leq k \leq r-1\right\}$, and so $k_{s}=k_{\ell}$.

\section{E. The group $G(r, r / 2,2), r / 2$ odd}

We use the notation from Section $2 \mathrm{~B}$, or from above for the group $G(r, r, 2)$. In this case, the group is not a real reflection group, hence $G(r, r / 2,2)$ acts by unitary matrices but not by orthogonal matrices.

Let $A$ be a graded Hecke algebra for $G(r, r / 2,2)$. The only conjugacy class for which $a_{g}$ can be nonzero is $\left\{t_{\xi_{1}^{k} \xi_{2}^{r / 2-k}(1,2)} \mid 0 \leq k<r\right\}$. Thus, in the algebra $A$,

$\left[v_{1}, v_{2}\right]=\beta \sum_{k}\left(t_{\xi_{1}^{2 k} \xi_{2}^{r / 2-2 k}(1,2)}-t_{\xi_{1}^{r / 2-2 k} \xi_{2}^{2 k}(1,2)}\right), \quad$ where $\quad \beta=a_{\xi_{2}^{r / 2}(1,2)}\left(v_{1}, v_{2}\right)$ 


\section{A different graded Hecke algebra for $G(r, 1, n)$}

The classification of graded Hecke algebras for complex reflection groups in Section 2 shows that there do not exist graded Hecke algebras $A \cong S(V) \otimes \mathbb{C} G$ for the groups $G=G(r, 1, n), r>2, n>3$. In this section, we define a different "semidirect product" of the symmetric algebra $S(V)$ and the group algebra $\mathbb{C} G$ for the groups $G(r, 1, n)$. These algebras are not graded Hecke algebras in the sense of Section 1, but they do have a structure similar to what we would expect from experience with graded Hecke algebras for real reflection groups. Is it possible that there is a general definition of graded Hecke algebras, different from that given in Section 1, which includes the algebras defined below as examples for the groups $G(r, 1, n)$ ?

We shall use the notation for the groups $G(r, 1, n)$ as in Section 2B so that the group $G(r, 1, n)$ is acting by monomial matrices on a vector space $V$ of dimension $n$ with orthonormal basis $\left\{v_{1}, \ldots, v_{n}\right\}$. Let $s_{i}$ denote the permutation $(i, i+1) \in$ $G(r, 1, n)$.

Define $H_{r, 1, n}^{*}$ to be the algebra generated by the group algebra $\mathbb{C} G(r, 1, n)$ and $V$ with relations

$$
\begin{array}{ll}
v_{i} v_{j}=v_{j} v_{i}, & \text { for all } 1 \leq i, j \leq n, \\
t_{\xi_{i}} v_{j}=v_{j} t_{\xi_{i}}, & \text { for all } 1 \leq i, j \leq n, \\
t_{s_{i}} v_{k}=v_{k} t_{s_{i}}, & \text { if } k \notin\{i, i+1\}, \\
t_{s_{i}} v_{i+1}=v_{i} t_{s_{i}}+\sum_{\ell=0}^{r-1} t_{\xi_{i}^{\ell} \xi_{i+1}^{-\ell},}, & \text { for } 1 \leq i \leq n-1 .
\end{array}
$$

The following proposition establishes an "evaluation homomorphism" for the algebras $H_{r, 1, n}^{*}$ which is a generalization of the homomorphism in (4.5).

Proposition 5.2. Define elements $\bar{v}_{k}$ in the group algebra $\mathbb{C} G(r, 1, n)$ by setting $\bar{v}_{1}=0$ and

$$
\bar{v}_{k}=\frac{1}{r} \sum_{i<k} \sum_{0 \leq \ell \leq r-1} t_{\xi_{i}^{\ell} \xi_{k}^{-\ell}(i, k)}, \quad \text { for } 2 \leq k \leq n .
$$

Then there is a surjective algebra homomorphism

$$
\begin{array}{ccc}
H_{r, 1, n}^{*} & \longrightarrow & \mathbb{C} G(r, 1, n) \\
t_{g} & \longmapsto & t_{g} \\
v_{k} & \longmapsto & \bar{v}_{k}
\end{array}
$$

Proof. We must check that the defining relations (5.1) of $H_{r, 1, n}^{*}$ hold with the $v_{k}$ replaced by the $\bar{v}_{k}$. 
For each $1 \leq k \leq n$, let

$$
\bar{z}_{k}=\bar{v}_{1}+\cdots+\bar{v}_{k}=\frac{1}{r} \sum_{\substack{1 \leq i<j \leq k \\ 0 \leq \ell \leq r-1}} t_{\xi_{i}^{\ell} \xi_{j}^{-\ell}(i, j)} .
$$

Then, for each $k, \bar{z}_{k} \in Z(\mathbb{C} G(r, 1, k))$ since it is the sum of the elements of the conjugacy class of reflections $t_{\xi_{i}^{\ell} \xi_{j}^{-\ell}(i, j)}$ in $G(r, 1, k)$. So $\bar{z}_{k}$ commutes with $\bar{z}_{1}, \ldots, \bar{z}_{k}$ and therefore $\bar{z}_{1}, \ldots, \bar{z}_{n}$ commute. Since $\bar{v}_{k}=\bar{z}_{k}-\bar{z}_{k-1}$, it follows that $\bar{v}_{1}, \ldots, \bar{v}_{n}$ also commute.

If $m>k$ then $t_{\xi_{m}}$ clearly commutes with $\bar{z}_{k}$. If $m \leq k$ then $t_{\xi_{m}}$ commutes with $\bar{z}_{k}$ since $\bar{z}_{k} \in Z(G(r, 1, k))$. So $t_{\xi_{m}}$ commutes with $\bar{z}_{1}, \ldots, \bar{z}_{n}$ and hence with $\bar{v}_{1}, \ldots, \bar{v}_{n}$.

Since

$$
\begin{aligned}
t_{s_{k}} \bar{v}_{k} t_{s_{k}} & =t_{s_{k}}\left(\sum_{\substack{i<k \\
0 \leq \ell \leq r-1}} t_{\xi_{i}^{\ell} \xi_{k}^{-\ell}(i, k)}\right) t_{s_{k}}=\sum_{\substack{i<k \\
0 \leq \ell \leq r-1}} t_{\xi_{i}^{\ell} \xi_{k+1}^{-\ell}(i, k+1)} \\
& =\sum_{\substack{i<k+1 \\
0 \leq \ell \leq r-1}} t_{\xi_{i}^{\ell} \xi_{k+1}^{-\ell}(i, k+1)}-\sum_{0 \leq \ell \leq r-1} t_{\xi_{k}^{\ell} \xi_{k+1}^{-\ell}(k, k+1)} \\
& =\bar{v}_{k+1}-\sum_{0 \leq \ell \leq r-1} t_{\xi_{k}^{\ell} \xi_{k+1}^{-\ell}} t_{s_{k}},
\end{aligned}
$$

it follows that

$$
\begin{aligned}
\bar{v}_{k} t_{s_{k}} & =t_{s_{k}} \bar{v}_{k+1}-\sum_{0 \leq \ell \leq r-1} t_{s_{k}} t_{\xi_{k}^{\ell} \xi_{k+1}^{-\ell}} t_{s_{k}}=t_{s_{k}} \bar{v}_{k+1}-\sum_{0 \leq \ell \leq r-1} t_{\xi_{k}^{-\ell} \xi_{k+1}^{\ell}} \\
& =t_{s_{k}} \bar{v}_{k+1}-\sum_{0 \leq \ell \leq r-1} t_{\xi_{k}^{\ell} \xi_{k+1}^{-\ell}} .
\end{aligned}
$$

\section{References}

[Bou] N. Bourbaki, Groupes et algebres de Lie, Chapt. IV-VI, Masson, Paris, 1981.

[Ca] R. W. Carter, Conjugacy classes in the Weyl group, Compositio Math. 25 (1972), 1-59.

[Dr] V. G. Drinfeld, Degenerate affine Hecke algebras and Yangians, Funct. Anal. Appl. 20 (1986), 58-60. 
[G+] M. Geck, G. Hiss, F. Lübeck, G. Malle and G. Pfeiffer, CHEVIE - A system for computing and processing generic character tables for finite groups of Lie type, Weyl groups and Hecke algebras, AAECC $\mathbf{7}$ (1996), 175-210.

$[\mathrm{Hu}]$ J. E. Humphreys, Reflection groups and Coxeter groups, Cambridge Studies in Advanced Mathematics 29, Cambridge University Press, Cambridge, 1990.

[Lu] G. Lusztig, Affine Hecke algebras and their graded version, J. Amer. Math. Soc. 2 (1989), $599-635$.

[Lu2] G. Lusztig, Cuspidal local systems and graded Hecke algebras I, Inst. Hautes Études Sci. Publ. Math. 67 (1988), 145-202.

[Mac] I. G. Macdonald, Symmetric functions and Hall polynomials, Second edition, Oxford Mathematical Monographs, Oxford University Press, New York, 1995.

[OS1] P. Orlik and L. Solomon, Coxeter arrangements, Proc. Symp. Pure Math. 40 (1983), 269-291.

[OS2] P. Orlik and L. Solomon, Arrangements defined by unitary reflection groups, Math. Ann. 261 (1982), 339-357.

[OT] P. Orlik and H. Terao, Arrangements of hyperplanes, Springer-Verlag, Berlin-Heidelberg, 1992.

[S+] M. Schönert et al., GAP - Groups, Algorithms, and Programming, Lehrstuhl D für Mathematik, Rheinisch Westfälische Technische Hochschule, Aachen, Germany, fifth edition, 1995.

[ST] G. C. Shephard and J. A. Todd, Finite Unitary Reflection Groups, Canad. J. Math. 6 (1954), 274-304.

[St1] R. Steinberg, Differential equations invariant under finite reflection groups, Trans. Amer. Math. Soc. 112 (1964), 392-400.

[St2] R. Steinberg, Endomorphisms of linear algebraic groups, Mem. Amer. Math. Soc. 80 (1968), 1-108.

Arun Ram

Department of Mathematics

University of Wisconsin

Madison, WI 53706

USA

e-mail: ram@math.wisc.edu
Anne V. Shepler

Department of Mathematics

University of North Texas

Denton, TX 76203

USA

e-mail: ashepler@unt.edu

(Received: July 25, 2001)

To access this journal online:

(20) http://www.birkhauser.ch 